\title{
The Effectiveness of Exercise Interventions for the Management of Frailty: A Systematic Review
}

\author{
Olga Theou, ${ }^{1}$ Liza Stathokostas, ${ }^{2}$ Kaitlyn P. Roland, ${ }^{1}$ Jennifer M. Jakobi, ${ }^{1}$ \\ Christopher Patterson, ${ }^{3}$ Anthony A. Vandervoort, ${ }^{4}$ and Gareth R. Jones ${ }^{1}$ \\ ${ }^{1}$ Human Kinetics, Faculty of Health and Social Development, University of British Columbia Okanagan, \\ Kelowna, BC, Canada V1V 1 V7 \\ ${ }^{2}$ School of Kinesiology, Faculty of Health Sciences, University of Western Ontario, London, ON, Canada N6A 3K7 \\ ${ }^{3}$ Division of Geriatric Medicine, McMaster University, Hamilton, ON, Canada L9C 7N4 \\ ${ }^{4}$ Graduate Program in Health and Rehabilitation Sciences, University of Western Ontario, London, ON, Canada N6G $1 H 1$
}

Correspondence should be addressed to Olga Theou, otheou@uwo.ca

Received 22 November 2010; Accepted 7 February 2011

Academic Editor: Eric Le Bourg

Copyright () 2011 Olga Theou et al. This is an open access article distributed under the Creative Commons Attribution License, which permits unrestricted use, distribution, and reproduction in any medium, provided the original work is properly cited.

\begin{abstract}
This systematic review examines the effectiveness of current exercise interventions for the management of frailty. Eight electronic databases were searched for randomized controlled trials that identified their participants as "frail" either in the title, abstract, and/or text and included exercise as an independent component of the intervention. Three of the 47 included studies utilized a validated definition of frailty to categorize participants. Emerging evidence suggests that exercise has a positive impact on some physical determinants and on all functional ability outcomes reported in this systematic review. Exercise programs that optimize the health of frail older adults seem to be different from those recommended for healthy older adults. There was a paucity of evidence to characterize the most beneficial exercise program for this population. However, multicomponent training interventions, of long duration ( $\geq 5$ months), performed three times per week, for 30-45 minutes per session, generally had superior outcomes than other exercise programs. In conclusion, structured exercise training seems to have a positive impact on frail older adults and may be used for the management of frailty.
\end{abstract}

\section{Introduction}

Frailty is an increasingly recognized geriatric syndrome that has a tremendous impact on the older individual, their family, and society as a whole. The terms "frail" and "frailty" are often used in the literature without clear definition or criteria [1]. Frailty is a complex concept and the precise definition remains to be elucidated. However, there is broad support for the understanding that frailty is a state of vulnerability, caused by multisystem reduction, ranging in severity from mild to severe, that places the individual at increased risk of adverse health outcomes $[2,3]$. The components of frailty are a mix of physiological, psychological, social, and environmental factors (e.g., sarcopenia, functional impairment, cognitive impairment, and depression). There is also a compelling need for effective interventions that manage frailty symptoms and as such, exercise may be the best medicine for this population.

Even though numerous operational (clinical) definitions of frailty were proposed to help develop screening criteria, there is not yet a standardized and valid method of clinically screening for frailty [4]. The most commonly used definitions of frailty are the frailty phenotype [5], the frailty index [6], the classification of frailty and vigorousness [7], and the Edmonton frail scale [8]. Fried et al. [5] proposed five indicators of physical frailty: muscle weakness, subjective fatigue, reduced physical activity, slow gait speed, and weight loss. Rockwood and Mitnitski's [6] frailty index is based on a mathematical model of the accumulation of deficits where a deficit can be any symptom, sign, disease, disability, 
and laboratory abnormality. In Speechley and Tinetti's [7] classification, older adults are classified as vigorous, transitional, or frail based on ten characteristics: age, gait/balance, walking activity for exercise, other physical activity for exercise, depression, use of sedatives, near-vision status, upper and lower extremity strength, and lower extremity disability. The Edmonton frail scale [8] proposes ten frailty indictors: cognition, self-rated health status, hospitalization, functional independence, social support, medication use, nutrition, mood, continence, and mobility.

Frailty should be treated in order to prevent the human and economic burden associated with this syndrome. Mounting evidence suggests that exercise interventions can be used to restore and/or maintain functional independence in older adults [9] and may potentially prevent, delay, or reverse the frailty process [10]. The American College of Sports Medicine's (ACSM) position stand [11] on exercise for older adults recommends that exercise prescription for frail people is more beneficial than any other intervention and that the contradictions to exercise for this population are the same as those used with younger and healthier people. In addition, the most recent updated ACSM guidelines [12] recommend that resistance and/or balance training should precede the aerobic training for this population. However, recommendations on the appropriate design of the exercise protocol were not included.

There are several systematic reviews published on the benefits of exercise in older adults [13-15]; however, to our knowledge, there are only two systematic reviews published specifically on the benefits of exercise in frail older adults $[16,17]$. A recent conceptual review on exercise and frailty concluded that exercise is the only intervention found to consistently improve sarcopenia, physical function, cognitive performance, and mood, which are important components of frailty [18]. Chin et al. [16] examined the effect of exercise on the functional ability of frail older adults. They included all studies that were published between 1995 and 2007, had identified their participants as frail either in the title or in the abstract, and focused only on functional outcomes. The authors concluded that exercise (resistance and multicomponent training) improved functional outcomes in this population. Daniels et al. [17] examined the effect of any type of intervention on disability in community-dwelling physically frail older adults. These investigators included studies that were published before 2007 and used at least one of the frailty indicators as described by Ferrucci et al. [3] (mobility, strength, endurance, nutrition, physical inactivity, balance, and motor processing) to identify their participants as frail but focused solely on disability. The presence of only one frailty indicator does not necessarily warrant that participants were frail since frailty is thought to be caused by multisystem reduction $[2,3]$. These researchers suggested that multicomponent exercise training (consisting of endurance, flexibility, balance, and resistance training) reduced disability impact, especially in moderately frail people. Seven additional articles have been published since 2007 measuring the effect of exercise on broad range of outcome measures of frail older adults in addition to functional ability and disability. An updated systematic review of exercise interventions for frail older people, that comprehensively examines how frailty is assessed and does not focus only on one specific outcome measure, has yet to be completed. The purpose of this systematic review was to consider the use of the term "frailty" in relation to exercise interventions and to examine the effectiveness of current exercise interventions for the management of frailty.

\section{Methods}

2.1. Literature Search. A literature search using multiple electronic bibliographic databases was conducted. Medline (OVID; 1950-), Embase (OVID; 1974-), Psycinfo (Scholars Portal; 1806-), Cinahl (OVID \& EBSCO; 1982-), Scopus (1823-), Ageline (AARP; 1978-), Eric (Proquest; 1966-), and SportDiscus (EBSCO; 1800-) were searched up to February 1, 2009. Reference lists of all relevant articles were cross-referenced by hand searching in order to identify additional articles. The primary search terms that were used for searching the electronic databases were frail and all reasonable expressions of exercise. The search strategy that was used for Medline is included in the appendix.

2.2. Inclusion/Exclusion Criteria. Studies met the following inclusion criteria: (1) acknowledged as a randomizedcontrolled trial, (2) full-text published in either English or French, (3) study participants were identified as "frail" in either the title, abstract, and/or text, (4) exercise was acknowledged as an independent component of the intervention. Physical activity was defined as any bodily movement produced by skeletal muscles that result in energy expenditure [19]. Exercise was defined as a form of physical activity that was structured and repetitive over an extended period of time, with the intention of improving fitness, performance or health [19]. Although frailty usually interacts with other chronic conditions, the purpose of this systematic review was to focus exclusively on frailty; therefore, those studies that targeted specific chronic disease conditions were excluded.

2.3. Data Collection and Analysis. The database search results were uploaded into a web-based system [20] which was used to manage the screening process. Duplicate citations were removed. To determine which studies would be included, two members of the review team independently screened the title and abstracts of the articles that were extracted from the literature search. The full text was retrieved electronically for studies that met reviewers' agreement based on the inclusion/exclusion criteria. For each article that satisfied these criteria, two reviewers independently extracted the following data: country that the study was conducted, number of participants in the intervention and control groups, age of participants at inclusion, sex of participants, living arrangements, inclusion criteria used to recruit participants, frailty definition that was used, characteristics of the exercise intervention (frequency, intensity, duration, and type), and outcome measures (Tables 1-4). Any disagreement on papers and data extracted between the two reviewers was resolved by a third reviewer. 
TABLE 1: Description of studies that were done in long-term care.

\begin{tabular}{|c|c|c|c|c|c|}
\hline $\begin{array}{l}N(\% \mathrm{~F}) \\
\text { Mean age }\end{array}$ & Inclusion criteria & Intervention characteristics & Outcome measures ${ }^{\mathrm{e}}$ & Quality & Reference \\
\hline $\begin{array}{l}194(71) \\
81\end{array}$ & $\begin{array}{l}>60 \text {, living in LTC and } \\
\text { experiencing ADL } \\
\text { disability }^{\mathrm{d}}\end{array}$ & $\begin{array}{l}\text { Multicomponent one-on-one } \\
\text { training (physical therapy), } 16 \\
\text { weeks, 3/week, } \\
\text { 30-45 min/session }\end{array}$ & $\begin{array}{l}\text { Cognition (MMSE), depression (Geriatric } \\
\text { Depression Scale); test batteries (physical } \\
\text { disability index); ADL disability (Katz } \\
\text { ADL); QOL (sickness impact profile); } \\
\text { utilization of resources (health care cost) }\end{array}$ & 5 & {$[69]$} \\
\hline $\begin{array}{l}191(73) \\
85\end{array}$ & $\begin{array}{l}>65 \text {, living in LTC and } \\
\text { experiencing ADL } \\
\text { disability }^{\mathrm{d}}\end{array}$ & $\begin{array}{l}\text { High-intensity functional } \\
\text { multicomponent training } \\
\text { (resistance, balance, and } \\
\text { walking), } 12 \text { weeks, } 2 \text {-3/week, } \\
45 \mathrm{~min} / \text { session, } 8-12 \text { rep based } \\
\text { on } 1 \text { RM }\end{array}$ & $\begin{array}{l}\text { Muscle function (lower strength); walking } \\
\text { speed ( } 2.4 \text { meters test); balance (BBS); falls } \\
\text { (incident rate) }\end{array}$ & 5 & {$[45,46]$} \\
\hline $\begin{array}{l}190(84) \\
88\end{array}$ & $\begin{array}{l}\text { Nursing home residents, } \\
\text { incontinence }^{\mathrm{d}}\end{array}$ & $\begin{array}{l}\text { Functional multicomponent } \\
\text { training (aerobic, resistance), } 32 \\
\text { weeks, } 5 / \text { week, } 75 \% \text { of maximum } \\
\text { workload }\end{array}$ & $\begin{array}{l}\text { Biochemical status (lymphocyte } \\
\text { subpopulations); cardio (exercise HR); } \\
\text { muscle function (upper strength); PA } \\
\text { (motion sensors and staff observations); } \\
\text { mobility endurance (walked or wheeled } \\
\text { distance) }\end{array}$ & 5 & {$[70]$} \\
\hline $\begin{array}{l}97(84) \\
84\end{array}$ & $\begin{array}{l}>65 \text {, physically } \\
\text { restrained nursing home } \\
\text { residents, extremely } \\
\text { impaired both } \\
\text { cognitively and } \\
\text { physicallyd }\end{array}$ & $\begin{array}{l}\text { Mutlicomponent training } \\
\text { (aerobic, resistance, mobility, } \\
\text { and safety practice), } 9 \text { weeks, } \\
\text { 3/week, } 10 \% \text { increase/week }\end{array}$ & $\begin{array}{l}\text { Psychosocial state (safety score); muscle } \\
\text { function (upper strength and endurance); } \\
\text { flexibility (rowing ROM); mobility } \\
\text { endurance (walk time, wheel time); chair } \\
\text { rises ( } 30 \mathrm{sec} \text { ) }\end{array}$ & 3 & {$[68]$} \\
\hline $\begin{array}{l}30(50) \\
81\end{array}$ & $\begin{array}{l}\text { Living in LTC, mild } \\
\text { cognitive impairment }\end{array}$ & $\begin{array}{l}\text { Multicomponent training } \\
\text { (aerobic, resistance, balance, and } \\
\text { flexibility), } 4 \text { weeks }\end{array}$ & $\begin{array}{l}\text { Psychosocial state (behavioral problems } \\
\text { and use of antipsychotic and hypnotic } \\
\text { medications) }\end{array}$ & 1 & {$[81]$} \\
\hline $\begin{array}{l}20(75) \\
88\end{array}$ & $>65$, living in LTC $^{d}$ & $\begin{array}{l}\text { Multicomponent training } \\
\text { (resistance, flexibility), } 48 \text { weeks, } \\
\text { 3/week, } 60 \mathrm{~min} / \text { session, } 1 \times 5-2 \\
\times 10 \mathrm{rep}\end{array}$ & $\begin{array}{l}\text { Cognition (MMSE); TUG; balance (BBS); } \\
\text { test batteries (PPT) }\end{array}$ & 5 & {$[71]$} \\
\hline $\begin{array}{l}71 \\
82\end{array}$ & Living in LTC $^{\mathrm{d}}$ & $\begin{array}{l}\text { Multicomponent training } \\
\text { (aerobic, resistance, balance, } \\
\text { flexibility, and coordination), } 48 \\
\text { weeks, 2/week, 10-60 min }\end{array}$ & $\begin{array}{l}\text { Psychosocial state (class satisfaction); PA } \\
\text { (daily activity level); chair rises }\end{array}$ & 4 & {$[93]$} \\
\hline $\begin{array}{l}68(87) \\
80\end{array}$ & Living in LTC $^{d}$ & $\begin{array}{l}\text { Multicomponent training } \\
\text { (resistance, balance, flexibility, } \\
\text { and walking), } 16 \text { weeks, 3/week, } \\
45 \mathrm{~min} / \mathrm{session}\end{array}$ & $\begin{array}{l}\text { Muscle function (lower and upper } \\
\text { strength); flexibility (sit and reach, } \\
\text { shoulder flexion); walking speed ( } 7 \text { meters } \\
\text { test); TUG; stair climb ( } 3 \text { steps); balance } \\
\text { (BBS); ADL disability (FIM) }\end{array}$ & 5 & {$[95]$} \\
\hline $\begin{array}{l}100(63) \\
87\end{array}$ & $>75$, living in $\mathrm{LTC}^{\mathrm{d}}$ & $\begin{array}{l}\text { High-intensity progressive } \\
\text { resistance training of the hip and } \\
\text { knee extensors, } 10 \text { weeks, } \\
\text { 3/week, } 45 \mathrm{~min} / \text { session, } 3 \times 8 \text { at } \\
80 \% 1 \mathrm{RM}\end{array}$ & $\begin{array}{l}\text { Body composition (weight, muscle mass, } \\
\text { muscle fiber distribution); nutrition } \\
\text { (energy intake); biochemical (muscle } \\
\text { damage and regeneration, central nuclei, } \\
\text { IGF-1); muscle function (lower strength); } \\
\text { PA (activity monitor); walking speed (6.1 } \\
\text { meters test); stair climb (4 steps) }\end{array}$ & 3 & {$[49-51]$} \\
\hline $\begin{array}{l}22 \\
82\end{array}$ & $>70$, living in $\mathrm{LTC}^{\mathrm{d}}$ & $\begin{array}{l}\text { Progressive resistance training, } \\
10 \text { weeks, } 3 / \text { week, } 3 \times 8 \text { rep at } \\
40 \% \text { and } 80 \% 1 \mathrm{RM}\end{array}$ & $\begin{array}{l}\text { Muscle function (KE strength and } \\
\text { endurance); mobility endurance (6-minute } \\
\text { walking test); chair rises ( } 3 \text { times); stair } \\
\text { climb (4 steps); ADL disability (health } \\
\text { assessment questionnaire disability index } \\
\text { subscale) }\end{array}$ & 5 & {$[82]$} \\
\hline $\begin{array}{l}41(80) \\
81\end{array}$ & Living in LTC $^{\mathrm{d}}$ & $\begin{array}{l}\text { Resistance training with music, } \\
28 \text { weeks, 2/week, } 45 \mathrm{~min} / \text { session }\end{array}$ & $\begin{array}{l}\text { Cognition (MMSE), depression (Geriatric } \\
\text { Depression Scale); muscle function (grip } \\
\text { strength); flexibility (KE and KF, spinal } \\
\text { flexion ROM); chair rises; balance (postural } \\
\text { sway); ADL disability (Barthel Index) }\end{array}$ & 3 & {$[79]$} \\
\hline
\end{tabular}


TABLE 1: Continued.

\begin{tabular}{|c|c|c|c|c|c|}
\hline $\begin{array}{l}N(\% \mathrm{~F}) \\
\text { Mean age }\end{array}$ & Inclusion criteria & Intervention characteristics & Outcome measures $^{\mathrm{e}}$ & Quality & Reference \\
\hline $\begin{array}{l}25(76) \\
83\end{array}$ & Living in $\mathrm{LTC}^{\mathrm{d}}$ & $\begin{array}{l}\text { Progressive lower body resistance } \\
\text { training (aimed at improving } \\
\text { muscle power), } 10 \text { weeks, } \\
\text { 3/week, } 20-60 \mathrm{~min} / \text { session }\end{array}$ & $\begin{array}{l}\text { Muscle function (KE strength and power); } \\
\text { walking speed ( } 6 \text { meters test); chair rises } \\
(30 \mathrm{sec}) \text {; TUG }\end{array}$ & 3 & [94] \\
\hline $\begin{array}{l}21(90) \\
90\end{array}$ & Living in LTC $^{d}$ & $\begin{array}{l}\text { Resistance training of knee } \\
\text { extensors and flexors, } 12 \text { weeks, } \\
3 / \text { week, } 45 \mathrm{~min} / \text { session, } 3 \times 8 \text { rep } \\
\text { at } 50-80 \% 1 \mathrm{RM}\end{array}$ & $\begin{array}{l}\text { Biochemical status (inflammatory markers); } \\
\text { muscle function (KE and KF strength) }\end{array}$ & 2 & {$[83]$} \\
\hline $\begin{array}{l}278(68) \\
85\end{array}$ & $\begin{array}{l}\text { Frail and prefrail (Fried's } \\
\text { frailty phenotype) }\end{array}$ & $\begin{array}{l}\text { Functional walking, balance, } 20 \\
\text { weeks, } 2 / \text { week, } 90 \mathrm{~min} / \text { session }\end{array}$ & $\begin{array}{l}\text { Test Batteries (POMA, physical } \\
\text { performance score based on } 4 \text { tests); ALD } \\
\text { Disability (GARS); Falls (incident rate) }\end{array}$ & 5 & {$[80]$} \\
\hline $\begin{array}{l}27(100) \\
82\end{array}$ & $>70$, living in $\mathrm{LTC}^{\mathrm{d}}$ & $\begin{array}{l}\text { Visual feedback-based balance } \\
\text { training, } 4 \text { weeks, 3/week, } \\
20-30 \mathrm{~min} / \text { session }\end{array}$ & $\begin{array}{l}\text { PA (interview); balance (postural sway, } \\
\text { weights shifting, BBS); falls (incident rate, } \\
\text { fear of falling) }\end{array}$ & 3 & {$[47,48]$} \\
\hline $\begin{array}{l}32(78) \\
83\end{array}$ & Living in LTC $^{d}$ & $\begin{array}{l}\text { Treadmill walking training, } 24 \\
\text { weeks, } 1-3 / \text { week, } 50-70 \% \text { of the } \\
\text { maximum speed }\end{array}$ & $\begin{array}{l}\text { Neurological (auditory stimulus reaction } \\
\text { time); walking speed ( } 10 \text { meters test); } \\
\text { balance (one leg stance, functional reach); } \\
\text { falls (incident rate, time to first fall) }\end{array}$ & 2 & {$[87]$} \\
\hline $\begin{array}{l}30 \\
77\end{array}$ & $\begin{array}{l}>65 \text {, inactivity, ADL } \\
\text { disability }^{c}\end{array}$ & $\begin{array}{l}\text { Water training (resistance, } \\
\text { flexibility, activities of daily living } \\
\text { (ADL) exercises, and relaxation), } \\
24 \text { weeks, } 1-2 / \text { week, } \\
60 \mathrm{~min} / \text { session, intensity based } \\
\text { on Borg's RPE scale }\end{array}$ & ADL disability (FIM); QOL (SF-36) & 5 & {$[89]$} \\
\hline $\begin{array}{l}24(63) \\
78\end{array}$ & $\begin{array}{l}\text { Living in LTC and } \\
\text { experiencing ADL } \\
\text { disability }^{\mathrm{d}}\end{array}$ & $\begin{array}{l}\text { Whole body vibration training, } 6 \\
\text { weeks, 3/week }\end{array}$ & $\begin{array}{l}\text { Muscle function (lower and upper strength); } \\
\text { flexibility (back scratch, chair } \\
\text { sit-and-reach); chair rises ( } 30 \mathrm{sec} \text { ); TUG; } \\
\text { test batteries (POMA) }\end{array}$ & 5 & {$[84]$} \\
\hline $\begin{array}{l}145(100) \\
86\end{array}$ & Living in $\mathrm{LTC}^{\mathrm{d}}$ & $\begin{array}{l}\text { Exercise therapy using the } \\
\text { Takizawa Program, } 12 \text { weeks, } \\
\text { 3/week }\end{array}$ & $\begin{array}{l}\text { Flexibility (shoulder, knee, ankle dorsiflex } \\
\text { ROM); ADL disability (FIM) }\end{array}$ & 5 & {$[90]$} \\
\hline
\end{tabular}

${ }^{\mathrm{a}}$ Validated operational definition of frailty.

${ }^{c}$ At least one frailty indicator in the inclusion criteria.

${ }^{\mathrm{d}}$ No frailty indicators on the inclusion criteria.

eSignificant between-group differences are shown in bold.

1RM: one repetition maximum; ADL: activities of daily living; BBS: Berg balance scale: FIM, functional independence measure; GARS: Groningen activity restriction scale; HR: heart rate; IGF-I: insulin-like growth factor I; KE: knee extension; KF: knee flexion; LTC: long-term care; MMSE: minimental status exam; PA: physical activity; POMA: Tinetti performance-oriented mobility assessment; PPT: physical performance test; QOL: quality of life; rep: repetitions; ROM: range of motion; RPE: rating of perceived exertion; SF-36: medical outcomes survey short-form 36; TUG: timed up-and-go test.

The methodological quality of the included studies was evaluated by two reviewers using the Jadad methodological quality criteria scale [21]. The double blinding criterion for this scale was modified due to the inability to blind allocation of study participants to an exercise intervention. A study could receive a Jadad score of zero to five. Differences in rating between the two reviewers were resolved by a third reviewer. The questions used were:

(i) was the method of randomization described in the paper? (2 points)

(ii) where the outcome assessors blinded to treatment allocation? ( 2 points)

(iii) was there a description of withdrawals and dropouts? (1 point)
Although we included all published outcome measures, for reporting we grouped these measures into three areas.

(i) Physical and psychosocial determinants included body composition, nutrition status, biochemical status, cardiorespiratory function, muscle function, flexibility, physical activity participation, neurological and cognitive function, and psychosocial state.

(ii) Functional ability included mobility, balance, and functional performance test batteries.

(iii) Adverse health consequences included ADL (activities of daily living) disability, quality of life, falls, and utilization of resources.

Due to variability in participant and intervention characteristics, assessment tools used to diagnose frailty, and outcome measures used across studies, a meta-analysis could 
TABLE 2: Description of studies that included community-dwelling older adults.

\begin{tabular}{|c|c|c|c|c|c|}
\hline $\begin{array}{l}N(\% \mathrm{~F}) \\
\text { Mean age }\end{array}$ & Inclusion criteria & Intervention characteristics & Outcome measures $^{\mathrm{e}}$ & Quality & Reference \\
\hline $\begin{array}{l}188(80) \\
83\end{array}$ & $\begin{array}{l}\text { Moderate and severe } \\
\text { physical frail (walking } \\
\text { test and chair stand test), } \\
>75^{\mathrm{b}}\end{array}$ & $\begin{array}{l}\text { Multicomponent training } \\
\text { (resistance, balance, and } \\
\text { flexibility), } 24 \text { weeks, } 3 / \text { week, up } \\
\text { to } 60 \text { min/session, } 2 \times 10 \text { rep at } \\
\text { three levels of difficulty }\end{array}$ & $\begin{array}{l}\text { Walking speed ( } 3 \text { meters test); chair rises ( } 3 \\
\text { times); test batteries (POMA, PPT); ADL } \\
\text { disability ( } 8 \text { ADL scale, IADL scale); falls } \\
\text { (fear of falling); utilization of resources } \\
\text { (admission and days spent in nursing home) }\end{array}$ & 5 & [34-37] \\
\hline $\begin{array}{l}161(71) \\
79\end{array}$ & $\begin{array}{l}\text { Frail (reduced physical } \\
\text { activity and weight loss) }\end{array}$ & $\begin{array}{l}\text { Supervised group functional } \\
\text { multicomponent training } \\
\text { (aerobic, resistance, flexibility, } \\
\text { speed, coordination, and skills } \\
\text { training), } 17 \text { weeks, } 2 / \text { week, } \\
45 \text { min/session, intensity } 6-8 \text { on } \\
\text { a } 10 \text {-point perceived exertion } \\
\text { scale }\end{array}$ & $\begin{array}{l}\text { Body composition (weight, muscle mass, } \\
\text { fat mass, and bone mass); nutrition (energy } \\
\text { and carbohydrate intake, fat and protein } \\
\text { intake, haematological indicators, sensory } \\
\text { performance, and appetite); biochemical } \\
\text { (cellular immune response); neurological } \\
\text { (visual stimulus reaction time, } \\
\text { coordination); psychosocial state (social } \\
\text { involvement); Groningen fitness test for the } \\
\text { elderly (strength, flexibility, balance, block } \\
\text { transfer, and reaction time); test batteries } \\
\text { (functional performance based on six } \\
\text { performance tests); ADL disability } \\
\text { (self-reported ability to perform } 16 \text { ADL); } \\
\text { QOL (Dutch scale of subjective wellbeing } \\
\text { for older persons, self-rated health) }\end{array}$ & 4 & [22-29] \\
\hline $\begin{array}{l}155(79) \\
77\end{array}$ & $\begin{array}{l}\text { Frail (SPPB and the } \\
\text { indication of difficulty } \\
\text { with } A D L),>70^{b}\end{array}$ & $\begin{array}{l}\text { Class-based multicomponent } \\
\text { training (functional aerobic, } \\
\text { resistance, and flexibility), } 72 \\
\text { weeks, 3/week, } 75 \mathrm{~min} / \text { session }\end{array}$ & Only exercise compliance & 5 & {$[60]$} \\
\hline $\begin{array}{l}115(52) \\
83\end{array}$ & $\begin{array}{l}\text { Mild-to-moderate } \\
\text { physical frail (PPT, } \\
\text { difficulty with ADL, and } \\
\text { reduced peak aerobic } \\
\text { power) },>78^{b}\end{array}$ & $\begin{array}{l}\text { Multicomponent (physical } \\
\text { therapy, aerobic, and resistance), } \\
36 \text { weeks, } 3 / \text { week, } \\
20-60 \mathrm{~min} / \text { session, } 3 \times 8-12 \mathrm{rep} \\
\text { at } 85-100 \% 1 \mathrm{RM}, 15 \mathrm{~min} \text { at } \\
65-70 \% \mathrm{VO}_{2} \max \text { and } 3-5 \mathrm{~min} \\
85-90 \% \mathrm{VO}_{2} \max \end{array}$ & $\begin{array}{l}\text { Body composition (weight, muscle mass, fat } \\
\text { mass, and bone mass); nutrition (energy } \\
\text { intake); cardio (VO } \mathbf{V O}_{2} \text { max, cardiac output, } \\
\text { exercise HR and peak BP, resting HR and } \\
\text { BP,and left ventricular stroke work); } \\
\text { muscle function (lower and upper } \\
\text { strength); balance (one leg stance, BBS); } \\
\text { test batteries (PPT, functional status } \\
\text { questionnaire); ADL disability (The Older } \\
\text { American Resources and Services } \\
\text { Instrument); QOL (SF-36) }\end{array}$ & 5 & {$[30-33]$} \\
\hline $\begin{array}{l}96(60) \\
83\end{array}$ & $\begin{array}{l}\text { Frail (reduced physical } \\
\text { activity and weight loss), } \\
>75, \text { receiving home } \\
\text { service, age under } 75, \\
\text { body mass index } \\
<30 \mathrm{~kg} / \mathrm{m}^{2 \mathrm{~b}}\end{array}$ & $\begin{array}{l}\text { Multicomponent training } \\
\text { (aerobic, resistance, and } \\
\text { Qigong), } 12 \text { weeks, } 2 / \text { week, } \\
60 \mathrm{~min} / \text { session, } 60-80 \% \text { intensity }\end{array}$ & $\begin{array}{l}\text { Body composition (weight, muscle mass); } \\
\text { nutrition (energy intake); psychosocial state } \\
\text { (health belief model); muscle function } \\
\text { (lower and upper strength); walking speed } \\
\text { (10 meters test); chair rises ( } 30 \text { sec); TUG; } \\
\text { stair climb ( } 30 \text { sec); balance (one leg and } \\
\text { tandem stance, modified figure 8); ADL } \\
\text { disability (FIM, IAM) }\end{array}$ & 3 & {$[74]$} \\
\hline $\begin{array}{l}84(57) \\
83\end{array}$ & $\begin{array}{l}\text { Mild-to-moderate } \\
\text { physical frail (PPT), } \\
>78, \text { sedentary, living } \\
\text { independently but with } \\
\text { difficulty }\end{array}$ & $\begin{array}{l}\text { Low-intensity supervised } \\
\text { multicomponent training } \\
\text { (resistance, balance, flexibility, } \\
\text { body handling skills, speed of } \\
\text { reaction, and coordination), } 12 \\
\text { weeks, } 3 / \text { week, three levels of } \\
\text { difficulty for each exercise }\end{array}$ & $\begin{array}{l}\text { Neurological (visual stimulus reaction time, } \\
\text { light touch and pressure sensation and } \\
\text { proprioception, coordination); muscle } \\
\text { function (lower and upper strength); } \\
\text { flexibility (shoulder, hip, knee, trunk } \\
\text { ROM); walking speed; balance (one leg } \\
\text { stance, obstacle course, BBS); test batteries } \\
\text { (PPT) }\end{array}$ & 2 & {$[64]$} \\
\hline $\begin{array}{l}77(81) \\
81\end{array}$ & $\begin{array}{l}\text { Physical frail (at least } \\
\text { one fall during the last } \\
\text { year and used some kind } \\
\text { of walking aid either } \\
\text { indoors or outdoors), } \\
>75^{\text {b }}\end{array}$ & $\begin{array}{l}\text { Functional multicomponent } \\
\text { training (resistance, balance), } 12 \\
\text { weeks, 2/week, } 40 \mathrm{~min} / \mathrm{session}\end{array}$ & $\begin{array}{l}\text { Psychosocial state (satisfaction); PA } \\
\text { (interview, frequency and duration of } \\
\text { outdoor walks); walking speed ( } 3 \text { meters } \\
\text { test); QOL (SF-36) }\end{array}$ & 5 & {$[75]$} \\
\hline
\end{tabular}


TABle 2: Continued.

\begin{tabular}{|c|c|c|c|c|c|}
\hline $\begin{array}{l}N(\% \mathrm{~F}) \\
\text { Mean age }\end{array}$ & Inclusion criteria & Intervention characteristics & Outcome measures $^{\mathrm{e}}$ & Quality & Reference \\
\hline $\begin{array}{l}53(100) \\
82\end{array}$ & $\begin{array}{l}\text { Frail (reduced physical } \\
\text { activity and unable to } \\
\text { get outdoors without } \\
\text { walking aids or help } \\
\text { from another person } \\
\text { and/or subjective } \\
\text { functional ability), >75, } \\
\text { receiving practical } \\
\text { and/or personal public } \\
\text { home care }^{\text {b }}\end{array}$ & $\begin{array}{l}\text { Home-based multicomponent } \\
\text { training (aerobic, resistance, } \\
\text { flexibility, and dynamic balance), } \\
20 \text { weeks, 3/week, } 26 \mathrm{~min} / \text { session }\end{array}$ & $\begin{array}{l}\text { Psychosocial state (mobility-tiredness } \\
\text { scale); muscle function (lower power and } \\
\text { upper strength); walking speed ( } 10 \text { meters } \\
\text { test); chair rises ( } 5 \text { times); balance (stance); } \\
\text { test batteries (PPT); QOL (EQ-5D } \\
\text { questionnaire, self-rated health status) }\end{array}$ & 3 & {$[77]$} \\
\hline $\begin{array}{l}424(69) \\
77\end{array}$ & $\begin{array}{l}70-89 \text {, inactivity, risk for } \\
\text { major mobility disability } \\
\text { as indicated by a } \\
\text { summary score of } \leq 9 \text { on } \\
\text { the SPPB (balance, } \\
\text { mobility, strength) }\end{array}$ & $\begin{array}{l}\text { Multicomponent training } \\
\text { (aerobic, resistance, balance, } \\
\text { flexibility, and walking), } 48 \\
\text { weeks, } 1-3 / \text { week, } 60 \mathrm{~min} / \mathrm{session}, \\
\text { intensity based on Borg's RPE } \\
\text { scale }\end{array}$ & $\begin{array}{l}\text { Psychosocial state (self-efficacy for the } \\
400-m \text { walk, satisfaction with physical } \\
\text { function); PA (CHAMPS questionnaire) }\end{array}$ & 5 & {$[66]$} \\
\hline $\begin{array}{l}13(0) \\
75\end{array}$ & $\begin{array}{l}>70, \text { at risk for fall } \\
\text { (history of fall past year, } \\
\text { muscle weakness, } \\
\text { measurable gait or } \\
\text { balance impairment) }\end{array}$ & $\begin{array}{l}\text { Multicomponent training } \\
\text { (resistance, balance, and } \\
\text { walking), } 12 \text { weeks, 3/week, } \\
60 \mathrm{~min} / \text { session }\end{array}$ & Biochemical status (immune) & 3 & {$[61]$} \\
\hline $\begin{array}{l}46(59) \\
81\end{array}$ & $\begin{array}{l}\text { Referred by their general } \\
\text { practitioner and patients } \\
\text { who could not leave } \\
\text { their home by } \\
\text { themselves }^{\text {d }}\end{array}$ & $\begin{array}{l}\text { Multicomponent and } \\
\text { comprehensive training (aerobic, } \\
\text { resistance, balance, flexibility, } \\
\text { rhythm, and reaction), } 12 \text { weeks, } \\
\text { 2/week, } 60 \mathrm{~min} / \text { session }\end{array}$ & $\begin{array}{l}\text { Body composition (muscle and fat mass), } \\
\text { cardio }\left(\mathrm{VO}_{2} \text { max); muscle function (upper }\right. \\
\text { strength); walking speed (10 meters test); } \\
\text { balance (BBS); QOL (SF-36) }\end{array}$ & 2 & {$[41,42]$} \\
\hline $\begin{array}{l}100(50) \\
78\end{array}$ & $\begin{array}{l}\text { Frail (inability to } \\
\text { descend stairs step over } \\
\text { step without holding the } \\
\text { railing) }\end{array}$ & $\begin{array}{l}\text { Home-based resistance training, } \\
10 \text { weeks, 3/week }\end{array}$ & $\begin{array}{l}\text { Muscle function (lower strength); walking } \\
\text { speed (10 meters test); mobility endurance } \\
\text { (6-minute walk test); chair rises (lowest } \\
\text { height someone stands); balance (postural } \\
\text { sway, functional reach); test batteries } \\
\text { (mobility skills protocol); QOL (SF-36); } \\
\text { falls (fear of falling) }\end{array}$ & 5 & {$[65]$} \\
\hline $\begin{array}{l}31(35) \\
71\end{array}$ & Moderate frail $(\mathrm{PPT})^{\mathrm{c}}$ & $\begin{array}{l}\text { Resistance training, } 24 \text { weeks, } \\
\text { 3/week, } 60 \mathrm{~min} / \text { session, } 3 \times 8 \text { rep } \\
\text { based on } 1 \mathrm{RM}\end{array}$ & $\begin{array}{l}\text { Body composition (muscle mass, muscle } \\
\text { fiber distribution); biochemical (IGF-I); } \\
\text { muscle function (KE strength) }\end{array}$ & 2 & {$[62]$} \\
\hline $\begin{array}{l}21(100) \\
78\end{array}$ & $\begin{array}{l}>70, \text { SPPB score } 4-10 \\
\text { (balance, mobility, } \\
\text { strength) }\end{array}$ & $\begin{array}{l}\text { Progressive resistance training } \\
\text { (mobility task specific and one } \\
\text { component at the fastest possible } \\
\text { velocity), } 12 \text { weeks, } 3 / \text { week, } \\
30 \mathrm{~min} / \mathrm{session}, 3 \text { sets }\end{array}$ & $\begin{array}{l}\text { Muscle function (lower power); walking } \\
\text { speed ( } 2.4 \text { meters test); chair rises ( } 5 \\
\text { times); balance (one leg stance); test } \\
\text { batteries (SPPB) }\end{array}$ & 4 & {$[63]$} \\
\hline $\begin{array}{l}17(71) \\
82\end{array}$ & $\begin{array}{l}\text { mild to moderate } \\
\text { physical frail }(\mathrm{PPT} \text { and } \\
\text { difficulty with } \mathrm{ADL})^{\mathrm{b}}\end{array}$ & $\begin{array}{l}\text { Resistance training, } 24 \text { weeks, } \\
3 / \text { week, initially } 1-2 \times 6-8 \text { rep at } \\
65-75 \% 1 \text { RM and progressed to } \\
3 \times 8-12 \text { rep at } 85-100 \% 1 \text { RM }\end{array}$ & $\begin{array}{l}\text { Body composition (weight, muscle mass); } \\
\text { biochemical status (muscle protein } \\
\text { synthesis, TNF- } \alpha \text {, LPL protein content); } \\
\text { muscle function (lower and upper } \\
\text { strength) }\end{array}$ & 2 & {$[38-40]$} \\
\hline $\begin{array}{l}21(48) \\
80\end{array}$ & $\begin{array}{l}>65 \text {, Using the day care } \\
\text { facility } 2 \text { or more times } \\
\text { per week }^{\mathrm{d}}\end{array}$ & $\begin{array}{l}\text { Horse riding simulator training, } \\
12 \text { weeks, } 2 / \text { week, } \\
10-30 \mathrm{~min} / \text { session, speed of the } \\
\text { simulator based on the physical } \\
\text { activity of participants }\end{array}$ & $\begin{array}{l}\text { Walking Speed ( } 5 \text { meters test); TUG; } \\
\text { Balance (stance, spinal alignment, and } \\
\text { functional reach) }\end{array}$ & 2 & {$[86]$} \\
\hline \multicolumn{6}{|c|}{$\begin{array}{l}{ }^{\mathrm{b}} \text { Nonvalidated operational definition of frailty. } \\
{ }^{\mathrm{c} A t} \text { least one frailty indicator in the inclusion criteria. } \\
\text { dNo frailty indicators on the inclusion criteria. } \\
\text { eSignificant between-group differences are shown in bold. } \\
\text { 1RM: one repetition maximum; ADL: activities of daily living; BBS: Berg balance scale; BP: blood pressure; FIM: functional independence measure; HR: } \\
\text { heart rate; IADL: instrumental activities of daily living; IAM: instrumental activity measure; IGF-I: insulin-like growth factor I; KE: knee extension; LPL: } \\
\text { lipoprotein lipase; PA: physical activity; POMA: Tinetti performance-oriented mobility assessment; PPT: physical performance test; QOL: quality of life; rep: } \\
\text { repetitions; ROM: range of motion; RPE: rating of perceived exertion; SF-36: medical outcomes survey short-form 36; SPPB: short physical performance } \\
\text { battery; TNF- } \alpha \text { : tumor necrosis factor alpha; TUG: timed up-and-go test; } \mathbf{V O}_{2} \text { max: maximal oxygen uptake. }\end{array}$} \\
\hline
\end{tabular}


TABLE 3: Description of studies that were done in retirement homes and in mixed settings.

\begin{tabular}{|c|c|c|c|c|c|}
\hline $\begin{array}{l}N(\% \mathrm{~F}) \\
\text { Mean age }\end{array}$ & Inclusion criteria & Intervention characteristics & Outcome measures ${ }^{\mathrm{e}}$ & Quality & Reference \\
\hline $\begin{array}{l}311(94) \\
81\end{array}$ & $\begin{array}{l}\text { Transitionally frail } \\
\text { (Speechley and Tinetti's } \\
\text { classification of frailty } \\
\text { and vigorousness), living } \\
\text { in retirement home, } \\
>70 \text {, at least one fall } \\
\text { within the past year }\end{array}$ & $\begin{array}{l}\text { Tai Chi, } 48 \text { weeks, } 2 / \text { week, } 60 \mathrm{~min} \\
\text { and progress to } 90 \mathrm{~min} / \mathrm{session}\end{array}$ & $\begin{array}{l}\text { Body composition (weight, body mass } \\
\text { index); Cardio (resting HR and BP); } \\
\text { Walking Speed; Chair Rises ( } 3 \text { times); } \\
\text { Balance (one leg stance, functional reach, } \\
\text { picking up an object from the floor, } 360 \\
\text { turn, postural control); QOL (Sickness } \\
\text { Impact Profile, self-rated health); Falls } \\
\text { (incident rate, fear of falling) }\end{array}$ & 5 & {$[52-56]$} \\
\hline $\begin{array}{l}57(88) \\
84\end{array}$ & $\begin{array}{l}\text { Frail (age, comorbidity, } \\
\text { polypharmacy, and } \\
\text { prolonged stay in } \\
\text { retirement home) }\end{array}$ & $\begin{array}{l}\text { Multicomponent training } \\
\text { (resistance, balance, and } \\
\text { flexibility), } 36 \text { weeks, } 3 / \text { week, } \\
60 \mathrm{~min} / \text { session }\end{array}$ & $\begin{array}{l}\text { Body composition (body mass index, } \\
\text { muscle mass); nutrition (haematological } \\
\text { indicators, resting energy expenditure); } \\
\text { muscle function (KE power); walking speed } \\
\text { ( } 6 \text { meters test); chair rises ( } 5 \text { times); stair } \\
\text { climb ( } 3 \text { steps) }\end{array}$ & 3 & {$[78]$} \\
\hline $\begin{array}{l}551(86) \\
80\end{array}$ & $\begin{array}{l}\text { Living in retirement } \\
\text { home }^{\mathrm{d}}\end{array}$ & $\begin{array}{l}\text { Functional multicomponent } \\
\text { training (aerobic, resistance, } \\
\text { balance, flexibility, and } \\
\text { coordination), } 48 \text { weeks, 2/week, } \\
60 \mathrm{~min} / \text { session }\end{array}$ & $\begin{array}{l}\text { Neurologic (visual stimulus reaction time); } \\
\text { muscle function (KE strength); mobility } \\
\text { endurance (6-minute walk test); balance } \\
\text { (postural sway, maximal balance range, and } \\
\text { coordinated stability tests); falls (incident } \\
\text { rate) }\end{array}$ & 3 & {$[91]$} \\
\hline $\begin{array}{l}161(86) \\
82\end{array}$ & $\begin{array}{l}>65 \text {, living in retirement } \\
\text { home and experiencing } \\
\text { ADL disability }\end{array}$ & $\begin{array}{l}\text { Task-specific resistance training } \\
\text { (training in bed-and chair-rise } \\
\text { subtasks), } 12 \text { weeks, 3/week, } \\
60 \mathrm{~min} / \text { session }\end{array}$ & $\begin{array}{l}\text { Muscle function (lower, upper, trunk } \\
\text { strength); flexibility (trunk, arm, leg } \\
\text { ROM); chair rises (bed- and chair-rise } \\
\text { task); balance (trunk) }\end{array}$ & 3 & {$[67]$} \\
\hline $\begin{array}{l}49(92) \\
79\end{array}$ & $\begin{array}{l}\text { Living at community or } \\
\text { retirement home, KE } \\
\text { muscle weakness }{ }^{c}\end{array}$ & $\begin{array}{l}\text { Resistance training, } 10 \text { weeks, } \\
\text { 3/week, } 60 \mathrm{~min} / \mathrm{session}, 3 \times 4 \text { rep }\end{array}$ & $\begin{array}{l}\text { Muscle function (isometric knee strength); } \\
\text { walking speed ( } 20 \text { meters test); TUG; stair } \\
\text { climb (box-stepping); balance (parallel, } \\
\text { semitandem, and tandem stance); ALD } \\
\text { disability (GARS) }\end{array}$ & 4 & {$[43,44]$} \\
\hline $\begin{array}{l}34(85) \\
81\end{array}$ & $\begin{array}{l}\text { Living at community or } \\
\text { LTC }^{\mathrm{d}}\end{array}$ & $\begin{array}{l}\text { Walking exercises, balance } \\
\text { training, } 12 \text { weeks, } 2-3 / \text { week, } \\
40 \mathrm{~min} / \mathrm{session}\end{array}$ & $\begin{array}{l}\text { TUG; stair climb ( } 5 \text { steps); balance (one leg } \\
\text { stance, functional reach, manual } \\
\text { perturbation test, functional balance scale); } \\
\text { test batteries (POMA) }\end{array}$ & 3 & {$[88]$} \\
\hline \multicolumn{6}{|c|}{$\begin{array}{l}\text { aValidated operational definition of frailty. } \\
{ }^{b} \text { Non-validated operational definition of frailty. } \\
{ }^{c} \text { At least one frailty indicator in the inclusion criteria. } \\
{ }^{d} \text { No frailty indicators on the inclusion criteria. } \\
{ }^{\text {e}} \text { Significant between-group differences are shown in bold. } \\
\text { ADL: activities of daily living; BP: blood pressure; GARS: Groningen activity restriction scale; HR: heart rate; KE: knee extension; LTC: long-term care; POMA } \\
\text { Tinetti performance-oriented mobility assessment; QOL: quality of life; rep: repetitions; ROM: range of motion; TUG: timed up-and-go test. }\end{array}$} \\
\hline
\end{tabular}

not be satisfactorily performed. Meta-analysis should only be considered when a group of studies have sufficient homogeneity between participants, interventions, and outcomes to provide a meaningful summary. In accordance with the Cochrane library if there is substantial clinical diversity a qualitative approach combining studies is appropriate. Previous systematic reviews on exercise and frailty $[16,17]$ did not conduct a meta-analysis for similar reasons. Subgroup analysis was done to examine factors that may explain the variability of these results. The outcomes were stratified based on the participants' characteristics (mean age, sex, and living arrangements) if a current frailty definition was used in the study, the intervention characteristics (frequency, intensity, duration, and type), and the methodological quality. We report the percentage of those outcome measures that significantly improved due to the exercise interventions (Table 5).

\section{Results}

3.1. Description of Studies. The preliminary search yielded 2247 citations. After an initial screening of all titles and abstracts, 303 articles remained from which full text were obtained. Of these articles, 74 met the inclusion/exclusion criteria [22-95]. The interrater reliability using Kappa score was 0.73 during screening of titles and abstracts and 0.80 during screening of full-text articles. One article was identified by the hand searching the reference lists of all relevant articles and reviews [96]. Articles using the same participants and intervention were grouped as a single 
TABLE 4: Description of studies that included hospitalized older adults.

\begin{tabular}{|c|c|c|c|c|c|}
\hline $\begin{array}{l}(\% \mathrm{~F}) \\
\text { Mean age }\end{array}$ & Inclusion criteria & Intervention characteristics & Outcome measures ${ }^{\mathrm{e}}$ & Quality & Reference \\
\hline $\begin{array}{l}76(72) \\
85\end{array}$ & $\begin{array}{l}>70 \text {, acutely ill patients, } \\
\text { acutely bedridden or } \\
\text { with reduced mobility }\end{array}$ & $\begin{array}{l}\text { Multicomponent training } \\
\text { (intensive physiotherapy), } 48 \\
\text { weeks, 5/week, } 30 \mathrm{~min} / \text { session }\end{array}$ & $\begin{array}{l}\text { Body composition (weight, body mass } \\
\text { index, fat mass, and arm and calf } \\
\text { circumference); nutrition (energy and } \\
\text { protein intake, haematological indicators); } \\
\text { muscle function (grip strength); ADL } \\
\text { disability (Katz ADL) }\end{array}$ & 2 & {$[85]$} \\
\hline $\begin{array}{l}68(100) \\
83\end{array}$ & $\begin{array}{l}>75 \text {, admitted to a } \\
\text { geriatric ward of } \\
\text { primary-care health } \\
\text { center hospital for an } \\
\text { acute illness, difficulties } \\
\text { in mobility and balance, } \\
\text { and symptoms such as } \\
\text { dizziness, reported falls, } \\
\text { or difficulty to walk } \\
\text { independentlyc }\end{array}$ & $\begin{array}{l}\text { Multicomponent training } \\
\text { (resistance, functional exercises, } \\
\text { relaxation), } 10 \text { weeks, } 2 / \text { week, } \\
90 \mathrm{~min} / \text { session, } 2 \times 8-10 \text { rep }\end{array}$ & $\begin{array}{l}\text { Depression (Zung self-rating depression } \\
\text { scale; muscle function (lower isometric } \\
\text { strength); walking speed (10 meters test); } \\
\text { balance (BBS); ADL disability (Joensuu } \\
\text { classification); falls (incident rate); } \\
\text { utilization of resources (health care, social } \\
\text { welfare, and falls cost) }\end{array}$ & 3 & {$[57-59,96]$} \\
\hline $\begin{array}{l}57(100) \\
82\end{array}$ & $\begin{array}{l}>75 \text {, older adults } \\
\text { admitted to a hospital } \\
\text { due to a fall or with a } \\
\text { history of injurious fall } \\
\text { that required medical } \\
\text { treatment }^{\mathrm{d}}\end{array}$ & $\begin{array}{l}\text { Functional multicomponent } \\
\text { training (resistance, balance), } 12 \\
\text { weeks, 3/week, 70-90\% of } \\
\text { maximum workload }\end{array}$ & $\begin{array}{l}\text { Depression (Geriatric Depression Scale); } \\
\text { psychosocial state (emotional status); } \\
\text { muscle function (lower and upper } \\
\text { strength); PA (Questionnaire); walking } \\
\text { speed (15 meters test); chair rises ( } 3 \text { times); } \\
\text { TUG; stair climb (13 steps); balance } \\
\text { (stance, functional reach); test batteries } \\
\text { (POMA); ADL disability (Barthel Index and } \\
\text { the Lawton Index of IADL); falls (incident } \\
\text { rate, fear of falling, walking steadiness, } \\
\text { emotional instability, and behavioral } \\
\text { changes following a fall) }\end{array}$ & 5 & {$[76]$} \\
\hline $\begin{array}{l}243(53) \\
79\end{array}$ & $\begin{array}{l}\text { frail (Winograd's frailty } \\
\text { scale), }>65^{\mathrm{a}}\end{array}$ & $\begin{array}{l}\text { Home-based resistance training, } \\
10 \text { weeks, } 3 / \text { week, } 3 \times 8 \text { rep at } \\
60-80 \% 1 \mathrm{RM}\end{array}$ & $\begin{array}{l}\text { Psychosocial state (degree of fatigue); } \\
\text { muscle function (KE strength); walking } \\
\text { speed (4 meters test); TUG; balance (BBS); } \\
\text { ADL disability (Barthel Index and Adelaide } \\
\text { Activities Profile); QOL (SF-36); falls } \\
\text { (incident rate, time to first fall, and fear of } \\
\text { falling) }\end{array}$ & 5 & {$[92]$} \\
\hline $\begin{array}{l}71(0) \\
78\end{array}$ & $\begin{array}{l}>65 \text {, hypogonadal } \\
\text { recuperative care } \\
\text { patients, recent } \\
\text { functional decline }\end{array}$ & $\begin{array}{l}\text { Progressive resistance training, } \\
12 \text { weeks, } 3 / \text { week, } 3 \times 8 \text { rep at } \\
20 \% \text { and } 80 \% 1 \mathrm{RM}\end{array}$ & $\begin{array}{l}\text { Body composition (muscle mass); muscle } \\
\text { function (lower and upper strength); test } \\
\text { batteries (aggregate functional performance } \\
\text { test) }\end{array}$ & 5 & {$[73]$} \\
\hline $\begin{array}{l}29(17) \\
79\end{array}$ & $\begin{array}{l}>65 \text {, recent } \\
\text { illness-induced } \\
\text { functional decline }^{c}\end{array}$ & $\begin{array}{l}\text { Progressive resistance training, } \\
12 \text { weeks, } 3 \times 8 \text { rep at } 20 \% \text { and } \\
80 \% 1 \mathrm{RM}\end{array}$ & $\begin{array}{l}\text { Body composition (weight, muscle mass, } \\
\text { and fat mass); nutrition (energy intake); } \\
\text { muscle function (lower and upper } \\
\text { strength); test batteries (aggregate } \\
\text { functional performance test) }\end{array}$ & 5 & {$[72]$} \\
\hline
\end{tabular}

${ }^{a}$ Validated operational definition of frailty.

${ }^{\mathrm{c}}$ At least one frailty indicator in the inclusion criteria.

${ }^{\mathrm{d}}$ No frailty indicators on the inclusion criteria.

${ }^{\text {e}}$ Significant between-group differences are shown in bold.

1RM: one repetition maximum; ADL: activities of daily living; BBS: Berg balance scale; IADL: instrumental activities of daily living; KE: knee extension; PA: physical activity; POMA: Tinetti performance-oriented mobility assessment; QOL: quality of life; rep: repetitions; SF-36: medical outcomes survey short-form 36; TUG: timed up-and-go test.

study although multiple subsets of the data were published independently. Therefore, 75 published articles described 47 studies (Figure 1). Eleven studies published multiple articles with various outcomes $[22-59,96]$. For the purpose of this review, we state the number of studies, while the citation indicates all published articles associated with those studies.
All relevant articles were published after 1993 and the majority (85\%) were published after 2000 [22-60, 62-64, 66, $67,70-78,80-92,94,96]$. Nineteen of the selected studies $[30-40,49-56,60-73]$ were from the USA, 18 [22-29, 41$48,57-59,74-85,96]$ from European countries, five [86-90] from Japan, three [91-93] from New Zealand and Australia, 
TABLE 5: Percentage of outcome measures that improved due to the exercise interventions.

\begin{tabular}{|c|c|c|c|c|}
\hline & $\begin{array}{c}\text { Physical \& psychosocial } \\
\text { determinants }\end{array}$ & $\begin{array}{c}\text { Functional } \\
\text { ability }\end{array}$ & $\begin{array}{c}\text { Adverse health } \\
\text { consequences }\end{array}$ & Reference \\
\hline All studies & $60 \%$ & $71 \%$ & $39 \%$ & {$[22-96]$} \\
\hline \multicolumn{5}{|l|}{ Age } \\
\hline $71-79$ years & $43 \%$ & $48 \%$ & $23 \%$ & {$[22-29,43,44,60-63,65,66,72,73,84,89,92]$} \\
\hline $80-90$ years & $66 \%$ & $76 \%$ & $44 \%$ & $\begin{array}{l}{[30-42,45-59,64,67-71,74-83,85-} \\
88,90,91,93-96]\end{array}$ \\
\hline \multicolumn{5}{|l|}{ Sex } \\
\hline Women $>$ men & $61 \%$ & $73 \%$ & $39 \%$ & {$[22-60,63,64,66-71,74-80,82-85,87-96]$} \\
\hline Men > women & $53 \%$ & $54 \%$ & - & {$[61,62,65,72,73,81,86]$} \\
\hline \multicolumn{5}{|l|}{ Living arrangement } \\
\hline Long term care & $76 \%$ & $78 \%$ & $50 \%$ & {$[45-51,68-71,79-84,87,89,90,93-95]$} \\
\hline Community & $57 \%$ & $77 \%$ & $44 \%$ & {$[22-42,60-66,74,75,77,86]$} \\
\hline Retirement home & $41 \%$ & $53 \%$ & $40 \%$ & {$[52-56,67,78,91]$} \\
\hline Hospital care & $50 \%$ & $64 \%$ & $25 \%$ & {$[57-59,72,73,76,85,92,96]$} \\
\hline \multicolumn{5}{|l|}{ Include operational definition } \\
\hline Yes & $50 \%$ & $64 \%$ & $30 \%$ & {$[22-40,52-56,60,62-65,74,75,77,78,80,92]$} \\
\hline No & $68 \%$ & $75 \%$ & $48 \%$ & $\begin{array}{l}{[41-51,57-59,61,63,66-73,76,79,81-91,93-} \\
96]\end{array}$ \\
\hline \multicolumn{5}{|l|}{ Include moderate frail } \\
\hline Yes & $62 \%$ & $82 \%$ & $50 \%$ & {$[30-40,52-56,62,64,80]$} \\
\hline No & $60 \%$ & $68 \%$ & $36 \%$ & {$[22-29,41-51,57-61,63,65-79,81-96]$} \\
\hline \multicolumn{5}{|l|}{ Type of intervention } \\
\hline Multicomponent training & $58 \%$ & $75 \%$ & $40 \%$ & $\begin{array}{l}{[22-37,41,42,45,46,57-61,64,66,68-71,74-} \\
78,81,85,91,93,95,96]\end{array}$ \\
\hline Resistance training & $67 \%$ & $61 \%$ & $27 \%$ & $\begin{array}{l}{[38-40,43,44,49-} \\
51,62,63,65,67,72,73,79,82,83,92,94]\end{array}$ \\
\hline \multicolumn{5}{|l|}{ Frequency of intervention } \\
\hline 2/week & $51 \%$ & $67 \%$ & $35 \%$ & {$[22-29,41,42,52-59,74,75,79,80,86,91,93,96]$} \\
\hline 3/week & $62 \%$ & $72 \%$ & $39 \%$ & $\begin{array}{l}{[30-40,43,44,47-51,60-65,67-69,71,73,76-} \\
78,82-84,90,92,94,95]\end{array}$ \\
\hline \multicolumn{5}{|l|}{ Duration of intervention } \\
\hline $1-4$ months & $61 \%$ & $70 \%$ & $30 \%$ & $\begin{array}{l}{[22-29,41-51,57-59,61,63-65,67-69,72-} \\
76,81-84,86,88,90,92,94-96]\end{array}$ \\
\hline $5-18$ months & $59 \%$ & $74 \%$ & $52 \%$ & $\begin{array}{l}{[30-40,52-56,60,62,66,70,71,77-} \\
80,85,87,89,91,93]\end{array}$ \\
\hline \multicolumn{5}{|c|}{ Duration per session of intervention } \\
\hline $30-45$ minutes & $60 \%$ & $78 \%$ & $43 \%$ & {$[22-29,45-51,63,69,75,77,79,83,85,86,88,95]$} \\
\hline 60-90 minutes & $49 \%$ & $60 \%$ & $38 \%$ & {$[41-44,52-62,66,67,71,74,78,80,89,91,96]$} \\
\hline \multicolumn{5}{|l|}{ Methodological quality } \\
\hline 0-4 Jadad score & $60 \%$ & $69 \%$ & $33 \%$ & $\begin{array}{l}{[22-29,38-44,47-51,57-59,61-} \\
64,67,68,74,77-79,81,83,85-88,91,93,94,96]\end{array}$ \\
\hline 5 Jadad score & $60 \%$ & $72 \%$ & $42 \%$ & $\begin{array}{l}{[30-37,45,46,52-56,60,65,66,69-} \\
73,75,76,80,82,84,89,90,92,95]\end{array}$ \\
\hline
\end{tabular}

and two [94, 95] from Canada. The number of participants in the articles varied from 13 [61] to 551 [91] and a total of 4915 participants were included in this systematic review.

3.2. Participants Characteristics. Participants of all studies were older than 60 years. The mean age ranged from 71 [62] to 90 years [83], and the mean age of the participants in all the included studies was 81.5 years. Most (74.5\%) of the included participants were female. In six studies [47, 48, 57-59, 63, 76, 77, 90, 96], participants were only females and in two studies $[61,73]$ only males, three studies $[82,89,93]$ did not specify the sex of the participants, and all remaining studies $[22-46,49-56,60,62,64-72,74,75,78-$ $81,83-88,91,92,94,95]$ included both sexes (females 


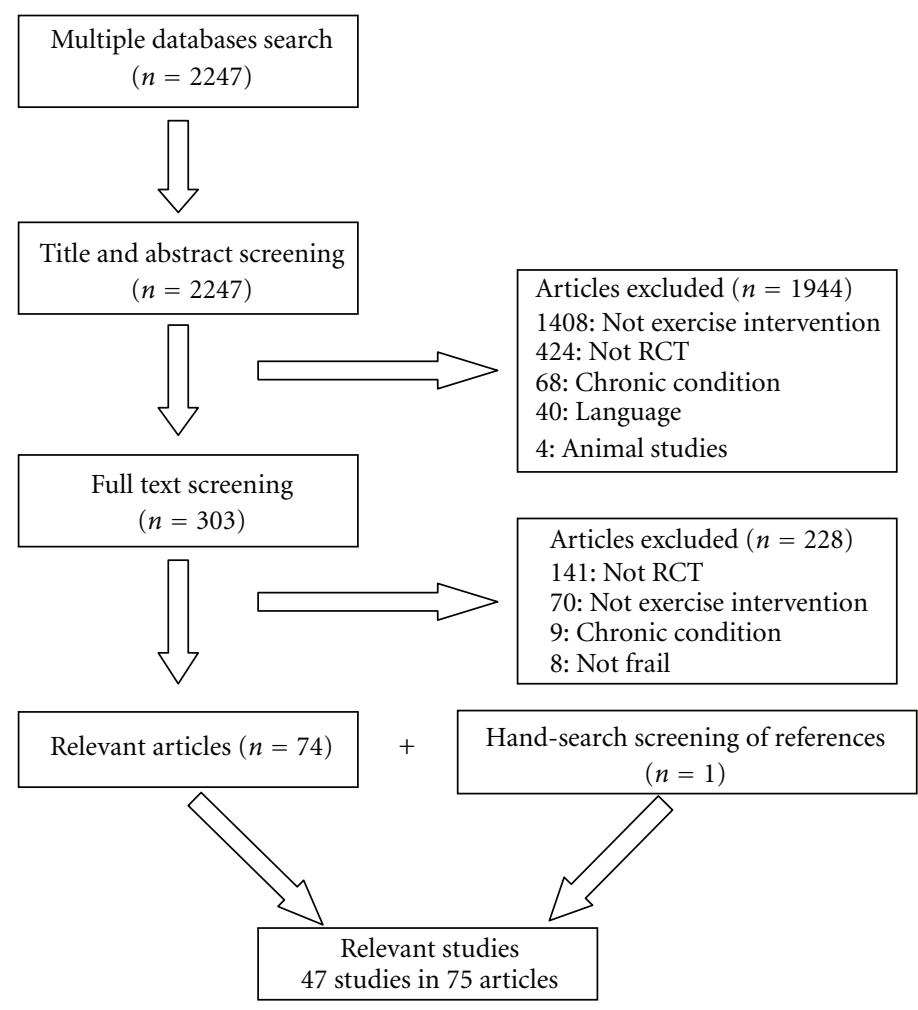

FIGURE 1: Flow chart of article screening. RCT: randomized controlled trial.

were the majority in most of them). There were too few studies, that measured adverse health consequences, where males were more abundant than females to suggest any sex differences in the effect of exercise on this outcome (Table 5) [65]. Nineteen studies [45-51, 68-71, 79-84, 87, 89, 90, 93-95] targeted those living in long-term care facilities (LTC) (Table 1), 16 [22-42, 60-66, 74, 75, 77, 86] focused on community-dwelling older adults (Table 2), one [88] included both community and LTC, four [52-56, 67, 78, 91] were conducted in retirement homes, one [43, 44] included both community and retirement homes (Table 3 ), and six $[57-59,72,73,76,85,92,96]$ involved hospital care (Table 4).

3.3. Measurement of Frailty. Even though in all studies the authors labeled their participants as "frail," only three studies utilized one of the validated operational definitions of frailty: Fried's frailty phenotype [80], Speechley and Tinetti's classification of frailty and vigorousness [52-56], and Winograd's frailty scale [92]. In 12 studies, nonvalidated definitions of frailty were used $[22-40,60,62,64,65,74,75$, $77,78]$. These studies used a variety of outcome measures in an assortment of combinations to measure frailty.

Most studies (32 studies) did not include an operational definition of frailty. Although nine studies mentioned at least one clinical marker of physical frailty (mobility and balance impairments, muscle weakness, testosterone deficiencies, and inactivity) in their inclusion criteria $[43,44,57-59,61$, $63,66,72,73,85,89,96]$, the inclusion criteria for the other
23 studies were living in LTC $[47-51,71,79,81-83,86-$ $88,90,93-95$ ], or retirement home [91], living in LTC or retirement home and experiencing ADL disability [45, 46, $67,69,84]$, nursing home residents with incontinence [70], physical restrained nursing home residents [68], patients who could not leave their home by themselves referred by general practitioners $[41,42]$, and older adults admitted to a hospital due to a fall or with a history of injurious falls that required medical treatment [76].

Earlier stages of frailty were included in seven studies. Five studies [30-33, 38-40,52-56, 62, 64] focused only on early stages of frailty whereas two studies $[34-37,80]$ included one group at an early stage and another group at a later stage of frailty. The early stage of frailty was measured using different tools and was classified differently across studies (prefrail [80], transitionally frail [52-56], mild-tomoderate frail $[30-33,38-40,64]$, and moderately frail [34$37,62])$. However, for the purpose of this review, we will call these early stages of frailty as moderate frailty.

\subsection{Intervention Characteristics}

3.4.1. Frequency. The majority of the exercise interventions were performed either twice (11 studies) [22-29, 41, 42, 52$59,74,75,79,80,86,91,93,96]$ or three times (27 studies) $[30-40,43,44,47-51,60-65,67-69,71,73,76-78,82-84$, $90,92,94,95]$ per week. Two studies increased the exercise frequency to five times per week $[70,85]$. Two other studies did not report exercise frequency $[72,81]$. 
3.4.2. Intensity. A detailed description of the exercise intensity was only provided for 18 of 47 studies [30-33, 38$40,49-51,63,67,68,70,72-74,76,80,82-84,87,91$, 92], and five studies [41, 42, 60, 77, 90, 93] offered no information regarding exercise intensity. In four studies, the exercise intensity was evaluated using a perceived exertion scale [22-29, 63, 66, 89]. Most of the interventions that utilized a resistance training program reported intensity as three sets of eight repetitions at approximately $80 \%$ of the individual's one repetition maximum (1RM) [38-40, 49$51,72,73,82,83,92]$. Three resistance training programs compared low-intensity (20 and 40\% 1RM) to high-intensity ( $80 \% 1 \mathrm{RM})$ training and found that the changes in muscle strength and endurance were greater in the high-intensity group compared with the low intensity $[72,73,82]$. However, improvements for functional ability were only marginally different, and ADL disability scores were similar between the two groups.

3.4.3. Duration. The duration of the interventions ranged from $1[47,48,81]$ to 18 months [60], and the most common duration was 3 months $[41,42,45,46,61,63,64,67$, $72-76,83,86,88,90]$. The duration per session ranged from 1084 to 90 minutes $[57-59,80,96]$, and the majority of the studies included interventions that lasted either 45 minutes/session (6 studies) [22-29, 45, 46, 49-51, 79, 83, 95] or 60 minutes/session (12 studies) $[41-44,52-56,61,62,66$, $67,71,74,78,89,91]$. Fourteen studies did not report the duration of the exercise sessions $[38-40,64,65,68,70,72$, $73,76,81,82,84,87,90,92]$.

3.4.4. Type. Twenty-four studies $[22-37,41,42,45,46,57-$ $61,64,66,68-71,74-78,81,85,91,93,95,96]$ included multicomponent exercise interventions (usually focusing on resistance, balance, aerobic, and flexibility training), 14 [38$40,43,44,49-51,62,63,65,67,72,73,79,82,83,92,94]$ resistance training, and seven other types of exercise interventions (walking exercise program [87], balance training $[47,48]$, water exercises [89], Tai Chi [52-56], whole body vibration exercise [84], exercise therapy using the Takizawa Program [90], and exercise using a horse-riding simulator [86]). However, each of these "other" exercise interventions were included only in one study; therefore, conclusions regarding their individual effect cannot be made (Table 5). In addition, two studies compared a walking with balance training and reported that their effect on functional ability and adverse health consequences was similar [80, 88].

3.5. Methodological Quality. The total Jadad methodological quality score of the studies ranged from 1 to 5 . Twenty-one studies [30-37, 45, 46, 52-56, 60, 65, 66, 69-73, 75, 76, 80, $82,84,89,90,92,95]$ had perfect scores, four [22-29, 43, $44,63,93]$ scored $4,13[47-51,57-59,61,67,68,74,77-$ $79,88,91,94,96]$ scored 3 , eight [38-42, 62, 64, 83, 85-87] scored 2, and one [81] scored 1. No studies were excluded on the basis of their quality score since one of the criteria of the scale was modified as described in the methods section. In 34 studies [22-37, 45-61, 65-67, 69-80, 82, 84, 88$92,94-96]$, the method of randomization was described whereas 13 studies [38-44, 62-64, 68, 81, 83, 85-87, 93] reported randomized-controlled trials, but the method of randomization was not described. Twenty-four studies [30$37,43-46,52-56,60,63,65,66,69-73,75,76,80,82,84$, $89,90,92,93,95]$ used a single-blinded design, two studies [22-29, 68] used designs where not all outcome assessors were blinded to treatment allocation, six studies [74, 77, 79, $87,88,91]$ were not blinded, and in 15 studies [38-42, 47$51,57-59,61,62,64,67,78,81,83,85,86,94,96]$, the authors did not include any information regarding blinding of the outcome assessors. All but one study [81] included a description of withdrawals and dropouts (Table 5).

3.6. Exercise Compliance, Adverse Events, and Cost. Thirty five of the 47 studies included information regarding exercise compliance $[22-37,41-59,63,66-69,71-80,82-84,88,89$, 91-96]. In these studies the compliance to exercise sessions (exercise classes attended) for the intervention groups ranged from $42 \%$ [91] to $100 \%$ [30-33], and the mean compliance was $84 \%$. From the seven studies [30-40, 52-56, 62, 64, 80] that included moderately frail adults only four [30-37, 52$56,80]$ reported exercise compliance rates, and these were similar to the other studies $(76 \%, 77 \%, 86 \%, 100 \%$, resp.).

In 16 studies, there were no adverse events during the period of the study or the adverse events were similar between the intervention and the control groups [22-29, 3437, 43-46, 49-59, 63, 69, 71, 76, 77, 79, 82, 93, 95, 96]. Latham et al. [92] reported that home-based high-intensity resistance exercise increased the risk of musculoskeletal injuries in frail people recently discharged from hospital. Eighteen out of 120 patients experienced episodes of back or knee pain that were directly attributable to the exercise. In another study that focused on moderately frail people, two out of 66 exercise participants experienced musculoskeletal injuries (rotator cuff injury and worsening of an existing shoulder problem during resistance training); however, there were no other adverse events reported [30-33]. In 29 studies, there was no discussion regarding adverse events during the period of the study $[38-42,47,48,60-62,64-68,70,72-$ 75, 78, 80, 81, 83-91, 94].

Three studies reported the cost of the exercise intervention [34, 57, 69]. Gill et al. [34] stated that six months of home-based physical therapy cost $\$ 1998$ (US) per participant. Mulrow et al. [69] reported that the cost per person for a 4-month exercise program in nursing homes was $\$ 1220$ (US) and for friendly visits (control) was $\$ 189$ (US). In another study [57], the cost per person (recently discharged from hospital) for the 10-week group exercise program was $\sim \$ 850$ (US) and for the home exercise program was $\sim \$ 2280$ (US).

\subsection{Outcome Measures}

\subsubsection{Physical and Psychosocial Determinants}

(1) Body Composition. Body composition was tested in 12 studies $[22,24-26,30-33,38,40,42,49,50,54,62,72-$ $74,78,85]$ using seven outcome measures; weight [2426, 30-32, 38, 40, 49, 54, 72, 74, 85], BMI [54, 78, 85], muscle 
mass [22, 24, 26, 31, 32, 38, 40, 42, 49, 62, 72-74, 78], muscle fiber distribution $[50,62]$, body fat mass $[26,31,42,72,85]$, bone mass [26, 33], and arm and calf circumference [85]. Each outcome measure was tested using various tools (e.g., DEXA and MRI). Exercise improved body composition only in nine of the 31 cases that body composition as an outcome was tested. Specifically weight increased in two [24-26, 72] of the eight studies, muscle mass in four $[22,24,26,31,32$, $38,40,49]$ of the 10 studies, and fat mass was reduced in one [72] of five studies. BMI, muscle fiber distribution, bone mass, and arm and calf circumference did not improve in any study.

(2) Nutritional Status. Nutritional status was reported in seven studies $[22,24,25,28,29,31,49,72,74,78,85]$ and evaluated using two- to three-week daily food records to calculate energy, protein, carbohydrate and fat intake $[22,24,25,28,31,49,72,74,85]$, haematological indicators of nutritional status $[22,25,28,29,78,85]$, resting energy expenditure [78], and sensory (smell, taste, and hunger perception) performance and appetite [22, 24]. Exercise improved dietary intake in three $[22,24,49,85]$ of the six studies, protein intake in one [85] of two studies, hematological indicators in one [85] of three studies, and carbohydrate intake in the only study [24] where this outcome was tested. Fat intake, resting energy expenditure, blood nutrient, sensory performance, and appetite did not change in the single study that evaluated these outcomes.

(3) Biochemical Status. Biochemical status was tested in seven studies measuring immune parameters (e.g., tumor necrosis factor alpha expression and interleukin) [29, 40, $61,70,83$ ], serum insulin-like growth factor-I (IGF-I) [51, $62]$, markers of muscle regeneration and damage $[50,51]$, and muscle protein metabolism [38-40]. Exercise improved immune status in two studies [29, 40], did not have an impact on two other studies [70,83], and had a negative impact (decreasing natural killer cell activity) in one study [61]. IGF-I improved in one of two studies where this outcome was measured [51]. Exercise improved markers of muscle regeneration $[50,51]$ and muscle protein synthesis [38-40], whereas markers of muscle damage were similar between the exercise and the control group [50,51].

(4) Cardiorespiratory Function. Cardiorespiratory function was tested in four studies [30, 32, 41, 42, 54, 70] using measurements of maximal oxygen consumption $\left(\mathrm{VO}_{2} \mathrm{max}\right)$ $[30,32,41,42]$, resting and exercise heart rate $[32,54$, 70], blood pressure [32,54], cardiac output [32], and left ventricular stroke volume [32]. Exercise had a significant impact on nine of the 11 studies that tested cardiorespiratory function. The only outcomes that did not change were resting heart rate and blood pressure in one [32] of the two studies $[32,54]$, where these outcomes were measured.

(5) Muscle Function. Muscle function was tested in 29 studies using a variety of tests $[30,31,38,40-45,49,50$, $58,62-65,67,68,70,72-74,76-79,82-85,91,92,94,95]$.
Thirteen studies $[42-45,49,50,58,62,63,65,78,82,83,91$, $92,94]$ tested only lower body muscle function, five studies $[41,42,68,70,79,85]$ examined only upper body muscle function, 10 studies [30, 31, 38, 40, 64, 72-74, 76, 77, 84, 95] investigated both lower and upper body muscle function, and one study [67] evaluated both lower and upper body and trunk muscle function. Four studies [63, 77, 78, 94] measured leg extension power, and one study [82] included leg extension endurance. The remaining studies tested only muscle strength. Various muscles of the upper body and lower body were tested using different tasks (e.g., 1RM, isometric, and isokinetic), and the most common muscles tested were the knee extension muscles.

Exercise training improved muscle function in the majority of studies. Only seven $[67,72,77,78,84,91,92]$ of the 24 studies that measured lower body muscle function and three $[67,76,84]$ of the 16 studies that measured upper body function did not show positive results, and the only study [67] that measured trunk strength was positive. Two of the four studies that measured leg extension power observed positive results $[63,94]$. Similarly, knee extension endurance was shown to improve in the one study where it was measured [82].

(6) Flexibility. Flexibility was examined in seven studies $[64,67,68,79,84,90,95]$ by using various tests (range of motion around various joints [64, 67, 68, 79, 90, 95], back scratch test [84], and sit and reach test $[84,95])$. Two studies $[64,67]$ measured lower and upper body and trunk flexibility, three studies [84, 90, 95] measured lower and upper body flexibility, one study [79] measured lower body and trunk flexibility, and one study [68] measured only upper body flexibility. Exercise improved flexibility in the majority of the studies that measured this outcome. Lower body flexibility improved in four $[64,67,90,95]$ of the six studies [64, 67, 79, 84, 90, 95], upper body flexibility improved in five $[64,67,68,90,95]$ of the six studies $[64,67,68,84,90,95]$, and trunk flexibility improved in all three studies $[64,67,79]$ that measured this outcome.

(7) Physical Activity Participation. Levels of physical activity participation were assessed in seven studies $[48,49,66,70$, $75,76,93]$ using activity monitors (motion sensors [70] and large-scale integrated activity monitors [49]) interviews [48, 75], questionnaires [66, 76, 93], and staff observations [70]. Exercise improved poststudy daily physical activity levels in all seven studies that measured this outcome regardless of how it was measured.

(8) Neurological and Cognitive Function. Neurological function was tested in four studies using visual stimulus reaction time $[28,64,91]$, auditory stimulus reaction time [87], coordination [28, 64], and peripheral sensation (light touch, pressure sensation, and proprioception) [64]. Exercise improved neurological function in three of eight cases that neurological function as an outcome was tested. More specifically exercise improved reaction time to visual stimulus in one [91] of the three studies, coordination in one [64] of 
the two studies, and reaction time to auditory stimulus in the only study that reported this outcome [87]. However, exercise did not affect peripheral sensation [64]. Cognitive function was measured in three studies $[69,71,79]$ using the minimental status exam, and improvement as a result of the exercise intervention was reported in only one [71] of these studies.

(9) Psychosocial State. Depression was measured in four studies using the Geriatric Depression Scale $[69,76,79]$ and the Zung self-rating depression scale [59]. Exercise reduced depression in half of the studies that measured this outcome $[59,79]$. Other psychosocial state outcomes measured in ten studies were emotional status [76], behavioural problems [81], degree of fatigue [92], tiredness due to mobility problems [77], safety scores [68], social involvement [27], health belief model [74], and self-efficacy and satisfaction with exercise $[66,75,93]$. Exercise had a positive influence on reducing tiredness related to mobility problems [77], behavioural problems [81], safety scores [68], and selfefficacy and satisfaction with exercise $[66,75,93]$. Exercise did not have an impact on emotional status [76], social involvement [27], and on the health belief model [74]. Exercise was reported to negatively influence self-perceived fatigue [92].

\subsubsection{Functional Ability}

(1) Mobility. Walking speed was measured in 20 studies using 10 different tests either at usual or fast speed [35, 37, $41-45,49,54,58,63-65,74-78,86,87,92,94,95]$. The distance of the walking tests ranged from $2.4[45,63]$ to 20 meters $[43,44]$. The most common was the 10 -meter walk test which was used in six studies [42, 51, 58, 65, 74, 77, 87]. Walking speed improved with exercise in 14 of 20 studies that measured this outcome $[35,37,41-43,45,49,54,58$, $63,65,75-77,86,94]$. Mobility endurance was tested using the 6-minute walking test in three studies $[65,81,82]$, and by measuring the distance, an individual could walk or move their wheel chairs during a standardized protocol in two studies $[68,70]$. Exercise improved walking endurance in three of the five studies that measured this outcome $[70,82$, 91]. Wheeling endurance improved in both studies $[68,70]$. Mobility using the timed up-and-go test was measured in 10 studies $[43,44,71,74,76,84,86,88,92,94,95]$ of which seven $[44,71,76,84,88,94,95]$ reported improved mobility. Chair-rising ability was tested in 15 studies using six different tests $[35,37,54,63,65,67,68,74,76-79,82,84,93,94]$. The most common tests were the three-repetition chair stands $[35,37,54,76,82]$ and the 30-second chair stands $[68,74,84,94]$, and both protocols were used in four studies. Exercise improved the chair-rising ability in 13 of 15 studies that measured this outcome $[35,37,54,63,65,67,74,76-$ 79, 82, 93, 94]. Stair-climbing ability was tested in eight studies using seven different protocols (e.g., number of steps, time, height, and power) [43, 44, 49, 74, 76, 78, 82, 88, 95]. Stair climbing ability improved in four of these studies [49, $74,76,82]$.
(2) Balance. Balance was measured in 22 studies [30, 41, 43$45,47,48,54,55,58,63-65,67,71,74,76,77,79,86-88$, $91,92,95]$ using multiple positions stance time tests (e.g., one leg stance, parallel stance, and semitandem stance) [30, $43,44,54,55,63,64,74,76,77,86-88]$, Berg balance scale $[30,41,45,47,48,58,64,71,92,95]$, functional reach test $[54,65,76,86-88]$, postural sway $[47,48,65,67,79,91]$, and nine other protocols that each was used only once $[47,48,54$, $55,64,74,86,88,91]$. Exercise improved 28 of the 41 balance outcomes that were tested. Balance measured using the Berg balance scale improved in all studies, with the exception of one [92].

(3) Functional Performance Test Batteries. In 15 studies, researchers used nine various test batteries to measure the functional performance of the participants $[23,29,30,35,37$, $63-65,69,71-73,76,77,80,84,88]$. The distribution of the functional performance test batteries across studies was as follows: Tinetti performance-oriented mobility assessment (POMA) [35, 37, 76, 80, 84, 88], physical performance test $[30,35,37,64,71,77]$, aggregate functional performance test $[72,73]$, and other tests used only once $[23,29,30,63,65,69$, 80]. Exercise improved the functional performance scores in 15 of 18 test batteries across 15 studies. All of the studies that used the physical performance test and the POMA reported positive changes associated with exercise.

\subsubsection{Adverse Health Consequences}

(1) ADL Disability. Activities of daily living (ADL) disability was measured in 16 studies $[23,30,34,35,37,43,44,69$, $74,76,79,80,82,85,89,90,92,95,96]$ using 13 scales; Functional Independence Measure [74, 89, 90, 95], Barthel Index $[76,79,92]$, Katz Activities of Daily Living Scale [65, 85], Groningen Activity Restriction Scale [43, 44, 80], and other scales used only once $[23,30,34,35,37,43,44,74,76$, $80,82,92,96]$. Exercise showed positive results in reducing ADL disability in seven of the 16 studies that measured this outcome [34, 35, 37, 79, 80, 82, 85, 89, 95]. More specifically, in the 10 studies $[34,35,65,74,76,79,85,89,90,92,95]$ that used basic ADL (BADL) scales only half $[34,35,79,85,89$, 95] showed positive effects, in the four studies [37, 74, 76, 92] that used IADL scales only one [37] improved IADL ability, and in the six studies $[23,30,43,44,80,82,96]$ that used both subscales only two $[80,82]$ reported significant improvements.

(2) Quality of Life. Quality of life was measured in ten studies [27, 30, 41, 42, 56, 65, 69, 75, 77, 89, 92] using five questionnaires: medical outcomes survey short-form 36 (SF36) questionnaire [30, 41, 42, 65, 75, 89, 92], self-rated health status $[27,56,77]$, sickness impact profile $[56,69]$, Dutch scale of subjective wellbeing for older persons [27], and EQ5D questionnaire [77]. Exercise improved quality of life in four $[30,41,42,75,89]$ of the 10 studies that measured this outcome. The questionnaire that was used in all positive studies was the SF-36. 
(3) Falls. Falls were examined in 11 studies [35, 46, 48, 52, $53,57,65,76,80,87,91,92]$ by testing falls incident rates during or following an intervention $[46,48,52,57,76,80$, $87,91,92]$, time to first fall $[87,92]$, fear of falling $[35,48,53$, $65,76,92]$, walking steadiness [76], and postfall emotional state [76]. Exercise reduced falls incidence in two $[48,91]$ of nine studies that measured this outcome whereas, in seven studies $[46,52,57,76,80,87,92]$, the risk for becoming a faller was similar between the exercise and the control group. Similarly, time to first fall was analogous between the exercise and control group in the two studies where it was measured $[87,92]$. Exercise had a positive impact on fear of falling in five of six studies $[35,48,53,65,76]$ and on the walking steadiness and post-fall emotional state, in the only study [76] where they were measured.

(4) Utilization of Resources. Utilization of resources was assessed in three studies [34, 57, 69]. Mulrow et al. [69] reported that there was no difference in health care charges (mean $\$ 11398$ (US) per person during the 4 months intervention) between the nursing home residents of the intervention and the control group. In another study, the health and social welfare costs and the fall-related health care costs were similar between the exercise and control groups (mean $\$ 12410$ (US) per person one year following the intervention) [57]. In addition, multicomponent training did not influence admission to a nursing home nor the number of days spent in a nursing home [34].

\section{Discussion}

This systematic review provides evidence that the term "frailty" was used extensively in relation to published exercise interventions. Most studies that examined the effect of exercise on frail people were published in the last decade and included primarily the oldest old ( $\geq 80$ years old) female participants. Only $32 \%$ of all studies included an operational definition of frailty, and from these studies only three (6\%) included a validated definition of frailty. Even among these studies, there was no agreement on the tools to measure frailty leading to large heterogeneity between the participants (e.g., various degrees of frailty). In most of the included studies, the participants were identified as frail, but no tools were used to diagnose frailty. As such, it is difficult to establish if indeed the participants of all studies were actually frail. In addition, there may be other studies with frail participants that were not included in this review as the authors did not identify their participants as frail. The most common exercise interventions for frail older adults included in this systematic review were multicomponent exercise programs performed three times per week for three months with each session lasting 60 minutes.

This systematic review provides evidence that exercise interventions have a positive impact on frail older adults. Even though the participants were frail, the exercise adherence was high and there were no adverse events in most reported studies, which support exercise as a safe and feasible intervention for this population. Exercise seems to benefit the oldest old, frail females more than younger frail males. This age-related difference may be explained by the fact that younger frail people may experience a ceiling effect on some outcome measures (BADL disability, mobility, balance, etc.). The sex-related difference may be explained by the fact that baseline physical and functional ability is less in females compared to males [97]; therefore, there is more room for exercise improvement by females.

Exercise seems to be more beneficial in frail people living in long-term care (LTC) facilities compared to the community. The evidence to support hospital and retirement homes exercise interventions is currently insufficient. However, these studies suggest that hospitalized frail older adults and those living in retirement homes do not seem to benefit from exercise to the same degree as that experienced by persons residing either the community or in LTC. Exercise may be more beneficial in one type of setting and not the other as a result of ceiling or floor effects on some outcome measures. For example, community dwelling frail adults are often relatively independent despite being frail, which would suggest that their ability to perform ADL would still be quite high. Therefore, exercise would not change ADL disability to the same degree as it would in those residing in LTC. In contrast, IADL will not change to the same degree in LTC populations as compared to community dwellers since their IADL ability would likely be too low to show a meaningful change.

In the studies where an operational definition of frailty was included, exercise seemed to be less effective in comparison to the studies that did not use definitions of frailty. Some of the studies that did not use a definition of frailty may have included people who were nonfrail; therefore, their participants were more likely to be healthier and perhaps more responsive to exercise training due to greater overload. In addition, exercise seems to be more effective in the earlier stages of frailty compared to the later stages of frailty. People with greater degree of frailty may not be able to exercise as long, as often, and as hard versus people at an earlier stage of frailty; therefore, they may not benefit from exercise to the same degree as the latter group.

Multicomponent training was more positive on the functional ability and adverse health consequences of the frail people; however, resistance training alone had a greater positive effect on the physical and psychosocial determinants. However, most of the physical and psychosocial determinants that the resistance training studies included involved muscle function outcomes. These outcomes had greater improvements if the exercise program focused solely on resistance training. Interventions lasting longer than five months seemed to result in greater gains on the adverse health consequences of the frail people than shorter duration interventions. Interventions with frequencies of three times per week were more beneficial for all outcomes, but the physical and psychosocial determinants showed the greatest changes. These differences likely occur because frail adults need more time to reach a level of exercise that may engender health and fitness benefit. In addition, longer duration interventions had more dropouts than shorter-duration interventions since many frail people would experience 
severe health problems and/or not survive to complete a long intervention; therefore, the results of the longer duration interventions are influenced by those survivors who are healthier. The duration for each session of exercise that seemed to be the most beneficial was $30-45$ minutes. This is less than what is usually recommended for healthy older adults [12] perhaps because frail people may fatigue easier. In addition, while frail people were able to exercise at higher intensities, low intensity exercise had a similar effect on the adverse health consequences.

None of the studies included in this systematic review used frailty as an outcome measure. The outcomes that were predominantly assessed were physical determinants and functional ability. There is good evidence that exercise improves cardiorespiratory function, muscle function, flexibility, physical activity participation, and functional ability of frail older adults. There is moderate evidence that exercise has a positive impact on psychosocial state, biochemical status, and adverse health consequences. Finally, there is little evidence to suggest that exercise positively influences body composition and nutritional status in frail people. Neurological and cognitive function and utilization of resources was not included as an outcome in a sufficient number of studies to make recommendations. Studies with perfect methodological quality (5 out of 5), in accordance with the Jaded criteria, had more favorable results than did lower quality studies. Those lower quality studies were likely more prone to bias (e.g., selection bias), which could make the exercise interventions less effective.

Our study is in agreement with the other systematic reviews that the most common exercise protocol for frail older adults is multicomponent training performed three times per week, and that there is good evidence to support exercise training for improving function, but the evidence is not as strong for improving ADL disability $[16,17]$. In addition, the exercise recommendations for a healthy older adult are likely going to be different from those targeting frail older adults. Specifically, frail older adults may need longterm exercise programs with shorter-duration sessions and a substantial balance component compared with the healthy older adults [12].

The results from the subgroup analysis (Table 5) showed that selected factors had an impact on the effect of exercise on the management of frailty and should be taken in consideration. For example, the improvements from exercise were greater when the frail participants were $80-90$ years compared with studies that included younger frail participants (71-79 years). Future studies examining the effect of exercise on frailty should consider these differences and not combine younger and older frail people within the same sample.

In conclusion, the recommendations made are based upon qualitative examination and should be interpreted with caution. Definitive conclusions regarding the beneficial effects of exercises intervention(s) on frailty should be determined with meta-analysis which was beyond the scope of this systematic review. Future systematic reviews should include only high-quality studies (e.g., RCT) and focus solely on specific outcomes. Although this will limit the number of studies included within the review, it will improve homogeneity making meta-analysis more feasible.

Future study in this area should also strive to use one of the existing validated definitions of frailty to assess participants prior to classifying them as frail. There is a genuine need for more high-quality studies on the effect of exercise on the psychosocial parameters and adverse health consequences. In addition, frailty should be used as an outcome measure in order to show if exercise can reverse frailty (frail reverse to nonfrail) or if older people can transition from a greater state of frailty to a lesser state of frailty with exercise. Future studies should also include larger sample sizes, participants with various degrees of frailty, and should examine age- and sex-related differences of the benefits of exercise in frail older adults. More studies are also needed with various training protocols (type, duration, frequency, and intensity) in order to determine the most beneficial and safe protocol for this population.

\section{Conclusion}

The term "frailty" has been used widely in relation to exercise. Structured exercise training has a positive impact on the frail older adults and should be used for the management of frailty. The most common exercise interventions that were summarized in this systematic review were multicomponent training interventions that lasted three months and were performed three times per week for one hour per session. However, longer-term multicomponent interventions with shorter-duration sessions (30-45 min) might be a better option for this population, especially for the prevention of adverse health consequences. More high-quality studies that use a validated definition of frailty, both as an inclusion criterion and as an outcome measure, and compare different participants' and interventions' characteristics are needed.

\section{Appendix}

\section{Medline Search}

(1) frail elderly/

(2) frail\$ or pre-frail\$ or prefrail\$

(3) or/1-2

(4) exercise/or exercise therapy/or exercise tolerance/or exercise test/

(5) physical fitness/or physical endurance/or physical therapy/

(6) rehabilitation/or therapeutics/

(7) sports/or weight lifting/or bicycling/or running/or swimming/or walking/

(8) leisure activities/or recreation/

(9) (physical adj3 (exercise\$ or therap\$ or conditioning or activit\$ or fitness))

(10) (exercise adj3 (train\$ or intervention\$ or protocol\$ or program\$ or therap\$ or activit\$)) 
(11) (fitness adj3 (train\$ or intervention\$ or protocol\$ or program $\$$ or therap $\$$ or activit $\$)$ )

(12) ((training or conditioning) adj3 (intervention\$ or protocol $\$$ or program $\$$ or activit $\$)$ )

(13) (rehabilitation adj3 (exercise\$ or train\$ or intervention\$ or protocol\$ or program\$ or therap\$ or activit\$))

(14) (therapeutic adj3 (exercise\$ or train\$ or intervention $\$$ or protocol\$ or program $\$$ or activit\$))

(15) (sport\$ or recreation\$ or leisure or cycl\$ or bicycl\$ or treadmill\$ or run\$ or swim\$ or walk\$)

(16) ((endurance or aerobic or cardio\$) adj3 (exercise\$ or fitness or train $\$$ or intervention $\$$ or protocol\$ or program $\$$ or therap\$ or activit\$))

(17) (muscle strengthening or progressive resist\$)

(18) ((weight or strength $\$$ or resistance or power) adj3 (exercise $\$$ or train\$ or lift\$))

(19) ((balance or flexibility) adj3 (exercise\$ or train\$ intervention $\$$ or protocol\$ or program\$ or activit\$))

(20) Tai Ji/or yoga/

(21) tai chi or yoga or pilates

(22) or/4-21

(23) 3 AND 22.

\section{Acknowledgments}

The authors thank Dr. John Feightner and Ms. Anh Tran for their assistance in writing this paper. No sources of funding were used to conduct this study and/or the preparation of the paper. The authors have no conflict of interests directly relevant to the content of this paper.

\section{References}

[1] M. Markle-Reid and G. Browne, "Conceptualizations of frailty in relation to older adults," Journal of Advanced Nursing, vol. 44, no. 1, pp. 58-68, 2003.

[2] D. B. Hogan, C. MacKnight, and H. Bergman, "Models, definitions, and criteria of frailty," Aging Clinical and Experimental Research, vol. 15, no. 3, pp. 1-29, 2003.

[3] L. Ferrucci, J. M. Guralnik, S. Studenski, L. P. Fried, G. B. Cutler Jr., and J. D. Walston, "Designing randomized, controlled trials aimed at preventing or delaying functional decline and disability in frail, older persons: a consensus report," Journal of the American Geriatrics Society, vol. 52, no. 4, pp. 625-634, 2004.

[4] O. Theou and M. Kloseck, "Tools to identify communitydwelling older adults in different stages of frailty," Physical and Occupational Therapy in Geriatrics, vol. 26, no. 3, pp. 1-21, 2008.

[5] L. P. Fried, C. M. Tangen, J. Walston et al., "Frailty in older adults: evidence for a phenotype," Journals of Gerontology A, vol. 56, no. 3, pp. M146-M156, 2001.

[6] K. Rockwood and A. Mitnitski, "Frailty in relation to the accumulation of deficits," Journals of Gerontology A, vol. 62, no. 7, pp. 722-727, 2007.
[7] M. Speechley and M. Tinetti, "Falls and injuries in frail and vigorous community elderly persons," Journal of the American Geriatrics Society, vol. 39, no. 1, pp. 46-52, 1991.

[8] D. B. Rolfson, S. R. Majumdar, R. T. Tsuyuki, A. Tahir, and K. Rockwood, "Validity and reliability of the edmonton frail scale," Age and Ageing, vol. 35, no. 5, pp. 526-529, 2006.

[9] D. H. Paterson, G. R. Jones, and C. L. Rice, "Aging and physical activity data on which to base recommendations for exercise in older adults," Applied Physiology, Nutrition, and Metabolism, vol. 32, no. 2F, supplement, pp. S75-S171, 2007.

[10] T. M. Gill, E. A. Gahbauer, H. G. Allore, and L. Han, "Transitions between frailty states among community-living older persons," Archives of Internal Medicine, vol. 166, no. 4, pp. 418-423, 2006.

[11] "American College of Sports Medicine position stand. Exercise and physical activity for older adults," Medicine \& Science in Sports \& Exercise, vol. 30, no. 6, pp. 992-1008, 1998.

[12] "American College of Sports Medicine position stand. Exercise and physical activity for older adults," Medicine \& Science in Sports \& Exercise, vol. 41, no. 7, pp. 1510-1530, 2009.

[13] R. Orr, J. Raymond, and M. F. Singh, "Efficacy of progressive resistance training on balance performance in older adults: a systematic review of randomized controlled trials," Sports Medicine, vol. 38, no. 4, pp. 317-343, 2008.

[14] J. G. Z. van Uffelen, A. Chin, M. J. Paw, M. Hopman-Rock, and W. van Mechelen, "The effects of exercise on cognition in older adults with and without cognitive decline: a systematic review," Clinical Journal of Sport Medicine, vol. 18, no. 6, pp. 486-500, 2008.

[15] P. G. Shekelle, M. Maglione, W. Mojica et al., Exercise Programs for Older Adults: A Systematic Review and Meta-Analysis, RAND Corporation Reprint Series, Centers for Medicare and Medicaid Services, Baltimore, Md, USA, 2003.

[16] A. Chin, M. J. Paw, J. G. Z. van Uffelen, I. Riphagen, and W. van Mechelen, "The functional effects of physical exercise training in frail older people: a systematic review," Sports Medicine, vol. 38, no. 9, pp. 781-793, 2008.

[17] R. Daniels, E. van Rossum, L. de Witte, G. I. Kempen, and W. van den Heuvel, "Interventions to prevent disability in frail community-dwelling elderly: a systematic review," BMC Health Services Research, vol. 8, article 278, 2008.

[18] F. Landi, A. M. Abbatecola, M. Provinciali et al., "Moving against frailty: does physical activity matter?" Biogerontology, vol. 11, no. 5, pp. 537-545, 2010.

[19] C. J. Casperson, K. E. Powell, and G. M. Christensen, "Physical activity, exercise, and physical fitness: definitions and distinctions for health-related research," Public Health Reports, vol. 100, no. 2, pp. 126-131, 1985.

[20] SRS 4.0, TrialStat Corporation, Ottawa, Ontario, Canada.

[21] A. R. Jadad, R. A. Moore, D. Carroll et al., "Assessing the quality of reports of randomized clinical trials: is blinding necessary?" Controlled Clinical Trials, vol. 17, no. 1, pp. 1-12, 1996.

[22] N. de Jong, "Sensible aging: using nutrient-dense foods and physical exercise with the frail elderly," Nutrition Today, vol. 36, no. 4, pp. 202-207, 2001.

[23] A. Chin, M. J. Paw, N. de Jong, E. G. Schouten, G. J. Hiddink, and F. J. Kok, "Physical exercise and/or enriched foods for functional improvement in frail, independently living elderly: a randomized controlled trial," Archives of Physical Medicine and Rehabilitation, vol. 82, no. 6, pp. 811-817, 2001.

[24] N. de Jong, A. Chin, M. J. Paw, C. de Graaf, and W. A. van Staveren, "Effect of dietary supplements and physical exercise on sensory perception, appetite, dietary intake and body 
weight in frail elderly subjects," British Journal of Nutrition, vol. 83, no. 6, pp. 605-613, 2000 .

[25] N. de Jong, A. Chin, M. J. Paw et al., "Functional biochemical and nutrient indices in frail elderly people are partly affected by dietary supplements but not by exercise," Journal of Nutrition, vol. 129, no. 11, pp. 2028-2036, 1999.

[26] N. de Jong, A. Chin, M. J. Paw, L. C. P. G. M. de Groot, G. J. Hiddink, and W. A. van Staveren, "Dietary supplements and physical exercise affecting bone and body composition in frail elderly persons," American Journal of Public Health, vol. 90, no. 6, pp. 947-954, 2000.

[27] A. Chin, M. J. Paw, N. de Jong, E. G. Schouten, W. A. van Staveren, and F. J. Kok, "Physical exercise or micronutrient supplementation for the wellbeing of the frail elderly? A randomised controlled trial," British Journal of Sports Medicine, vol. 36, no. 2, pp. 126-131, 2002.

[28] N. de Jong, A. Chin, M. J. Paw et al., "Nutrient-dense foods and exercise in frail elderly: effects on B vitamins, homocysteine, methylmalonic acid, and neuropsychological functioning," American Journal of Clinical Nutrition, vol. 73, no. 2, pp. 338-346, 2001.

[29] A. Chin, M. J. Paw, N. de Jong et al., "Immunity in frail elderly: a randomized controlled trial of exercise and enriched foods," Medicine and Science in Sports and Exercise, vol. 32, no. 12, pp. 2005-2011, 2000.

[30] E. F. Binder, K. B. Schechtman, A. A. Ehsani et al., "Effects of exercise training on frailty in community-dwelling older adults: results of a randomized, controlled trial," Journal of the American Geriatrics Society, vol. 50, no. 12, pp. 1921-1928, 2002.

[31] E. F. Binder, K. E. Yarasheski, K. Steger-May et al., "Effects of progressive resistance training on body composition in frail older adults: results of a randomized, controlled trial," Journals of Gerontology A, vol. 60, no. 11, pp. 1425-1431, 2005.

[32] A. A. Ehsani, R. J. Spina, L. R. Peterson et al., "Attenuation of cardiovascular adaptations to exercise in frail octogenarians," Journal of Applied Physiology, vol. 95, no. 5, pp. 1781-1788, 2003.

[33] D. T. Villareal, K. Steger-May, K. B. Schechtman et al., "Effects of exercise training on bone mineral density in frail order women and men: a radomised controlled trial," Age and Ageing, vol. 33, no. 3, pp. 309-312, 2004.

[34] T. M. Gill, D. I. Baker, M. Gottschalk, P. N. Peduzzi, H. Allore, and A. Byers, "A program to prevent functional decline in physically frail, elderly persons who live at home," The New England Journal of Medicine, vol. 347, no. 14, pp. 1068-1074, 2002.

[35] P. Peduzzi, Z. Guo, R. A. Marottoli, T. M. Gill, K. Araujo, and H. G. Allore, "Improved self-confidence was a mechanism of action in two geriatric trials evaluating physical interventions," Journal of Clinical Epidemiology, vol. 60, no. 1, pp. 94-102, 2007.

[36] T. M. Gill, D. I. Baker, M. Gottschalk et al., "A prehabilitation program for physically frail community-living older persons," Archives of Physical Medicine and Rehabilitation, vol. 84, no. 3, pp. 394-404, 2003.

[37] T. M. Gill, D. I. Baker, M. Gottschalk, P. N. Peduzzi, H. Allore, and P. H. Van Ness, "A prehabilitation program for the prevention of functional decline: effect on higherlevel physical function," Archives of Physical Medicine and Rehabilitation, vol. 85, no. 7, pp. 1043-1049, 2004.

[38] K. E. Yarasheski, J. Pak-Loduca, D. L. Hasten, K. A. Obert, M. B. Brown, and D. R. Sinacore, "Resistance exercise training increases mixed muscle protein synthesis rate in frail women and men $\geq 76$ yr old," American Journal of Physiology, vol. 277, no. 1, pp. E118-E125, 1999.

[39] J. N. Schulte and K. E. Yarasheski, "Effects of resistance training on the rate of muscle protein synthesis in frail elderly people," International Journal of Sport Nutrition and Exercise Metabolism, vol. 11, pp. S111-S118, 2001.

[40] J. S. Greiwe, C. Bo, D. C. Rubin, K. E. Yarasheski, and C. F. Semenkovich, "Resistance exercise decreases skeletal muscle tumor necrosis factor $\alpha$ in frail elderly humans," FASEB Journal, vol. 15, no. 2, pp. 475-482, 2001.

[41] C. H. Worm, E. Vad, L. Puggaard, H. Støvring, J. Lauritsen, and J. Kragstrup, "Effects of a multicomponent exercise program on functional ability in community-dwelling, frail older adults," Journal of Aging and Physical Activity, vol. 9, no. 4, pp. 414-424, 2001.

[42] H. Frederiksen, L. Bathum, C. Worm, K. Christensen, and L. Puggaard, "ACE genotype and physical training effects: a randomized study among elderly Danes," Aging Clinical and Experimental Research, vol. 15, no. 4, pp. 284-291, 2003.

[43] H. C. Boshuizen, L. Stemmerik, M. H. Westhoff, and M. Hopman-Rock, "The effects of physical therapists' guidance on improvement in a strength-training program for the frail elderly," Journal of Aging and Physical Activity, vol. 13, no. 1, pp. 5-22, 2005.

[44] M. H. Westhoff, L. Stemmerik, and H. C. Boshuizen, "Effects of a low-intensity strength-training program on knee-extensor strength and functional ability of frail older people," Journal of Aging and Physical Activity, vol. 8, no. 4, pp. 325-342, 2000.

[45] E. Rosendahl, N. Lindelöf, H. Littbrand et al., "High-intensity functional exercise program and protein-enriched energy supplement for older persons dependent in activities of daily living: a randomised controlled trial," Australian Journal of Physiotherapy, vol. 52, no. 2, pp. 105-113, 2006.

[46] E. Rosendahl, Y. Gustafson, E. Nordin, L. Lundin-Olsson, and L. Nyberg, "A randomized controlled trial of fall prevention by a high-intensity functional exercise program for older people living in residential care facilities," Aging Clinical and Experimental Research, vol. 20, no. 1, pp. 67-75, 2008.

[47] S. E. Sihvonen, S. Sipilä, and P. A. Era, "Changes in postural balance in frail elderly women during a 4-week visual feedback training: a randomized controlled trial," Gerontology, vol. 50, no. 2, pp. 87-95, 2004.

[48] S. Sihvonen, S. Sipilä, S. Taskinen, and P. Era, "Fall incidence in frail older women after individualized visual feedback-based balance training," Gerontology, vol. 50, no. 6, pp. 411-416, 2004.

[49] M. A. Fiatarone, E. F. O’Neill, N. D. Ryan et al., "Exercise training and nutritional supplementation for physical frailty in very elderly people," The New England Journal of Medicine, vol. 330, no. 25, pp. 1769-1775, 1994.

[50] M. A. Fiatarone Singh, W. Ding, T. J. Manfredi et al., "Insulinlike growth factor I in skeletal muscle after weight-lifting exercise in frail elders," American Journal of Physiology, vol. 277, no. 1, pp. E135-E143, 1999.

[51] M. L. Urso, M. A. Fiatarone Singh, W. Ding, W. J. Evans, A. C. Cosmas, and T. G. Manfredi, "Exercise training effects on skeletal muscle plasticity and IGF-1 receptors in frail elders," Age, vol. 27, no. 2, pp. 117-125, 2005.

[52] S. L. Wolf, R. W. Sattin, M. Kutner, M. O'Grady, A. I. Greenspan, and R. J. Gregor, "Intense Tai Chi exercise training and fall occurrences in older, transitionally frail adults: a randomized, controlled trial," Journal of the American Geriatrics Society, vol. 51, no. 12, pp. 1693-1701, 2003. 
[53] R. W. Sattin, K. A. Easley, S. L. Wolf, Y. Chen, and M. H. Kutner, "Reduction in fear of falling through intense tai chi exercise training in older, transitionally frail adults," Journal of the American Geriatrics Society, vol. 53, no. 7, pp. 1168-1178, 2005.

[54] S. L. Wolf, M. O'Grady, K. A. Easley, Y. Guo, R. W. Kressig, and M. Kutner, "The influence of intense Tai Chi training on physical performance and hemodynamic outcomes in transitionally frail, older adults," Journals of Gerontology A, vol. 61, no. 2, pp. 184-189, 2006.

[55] C. J. Hass, R. J. Gregor, D. E. Waddell et al., “The influence of Tai Chi training on the center of pressure trajectory during gait initiation in older adults," Archives of Physical Medicine and Rehabilitation, vol. 85, no. 10, pp. 1593-1598, 2004.

[56] A. I. Greenspan, S. L. Wolf, M. E. Kelley, and M. O'Grady, "Tai Chi and perceived health status in older adults who are transitionally frail: a randomized controlled trial," Physical Therapy, vol. 87, no. 5, pp. 525-535, 2007.

[57] L. Timonen, T. Rantanen, E. Mäkinen, T. E. Timonen, T. Törmäkangas, and R. Sulkava, "Cost analysis of an exercise program for older women with respect to social welfare and healthcare costs: a pilot study," Scandinavian Journal of Medicine and Science in Sports, vol. 18, no. 6, pp. 783-789, 2008.

[58] L. Timonen, T. Rantanen, O. P. Ryynänen, S. Taimela, T. E. Timonen, and R. Sulkava, "A randomized controlled trial of rehabilitation after hospitalization in frail older women: effects on strength, balance and mobility," Scandinavian Journal of Medicine and Science in Sports, vol. 12, no. 3, pp. 186-192, 2002.

[59] L. Timonen, T. Rantanen, T. E. Timonen, and R. Sulkava, "Effects of a group-based exercise program on the mood state of frail older women after discharge from hospital," International Journal of Geriatric Psychiatry, vol. 17, no. 12, pp. 1106-1111, 2002.

[60] J. A. Schmidt, C. Gruman, M. B. King, and L. I. Wolfson, "Attrition in an exercise intervention: a comparison of early and later dropouts," Journal of the American Geriatrics Society, vol. 48, no. 8, pp. 952-960, 2000.

[61] H. G. Rincón, G. F. Solomon, D. Benton, and L. Z. Rubenstein, "Exercise in frail elderly men decreases natural killer cell activity," Aging Clinical and Experimental Research, vol. 8, no. 2, pp. 109-112, 1996.

[62] J. V. Hennessey, J. A. Chromiak, S. Della Ventura et al., "Growth hormone administration and exercise effects on muscle fiber type and diameter in moderately frail older people," Journal of the American Geriatrics Society, vol. 49, no. 7, pp. 852-858, 2001.

[63] J. F. Bean, S. Herman, D. K. Kiely et al., "Increased velocity exercise specific to task (InVEST) training: a pilot study exploring effects on leg power, balance, and mobility in community-dwelling older women," Journal of the American Geriatrics Society, vol. 52, no. 5, pp. 799-804, 2004.

[64] M. Brown, D. R. Sinacore, A. A. Ehsani, E. F. Binder, J. O. Holloszy, and W. M. Kohrt, "Low-intensity exercise as a modifier of physical frailty in older adults," Archives of Physical Medicine and Rehabilitation, vol. 81, no. 7, pp. 960-965, 2000.

[65] J. M. Chandler, P. W. Duncan, G. Kochersberger, and S. Studenski, "Is lower extremity strength gain associated with improvement in physical performance and disability in frail, community-dwelling elders?" Archives of Physical Medicine and Rehabilitation, vol. 79, no. 1, pp. 24-30, 1998.

[66] W. J. Rejeski, A. C. King, J. A. Katula et al., "Physical activity in prefrail older adults: confidence and satisfaction related to physical function," Journals of Gerontology B, vol. 63, no. 1, pp. P19-P26, 2008.

[67] N. B. Alexander, A. T. Galecki, M. L. Grenier et al., "Taskspecific resistance training to improve the ability of activities of daily living-impaired older adults to rise from a bed and from a chair," Journal of the American Geriatrics Society, vol. 49, no. 11, pp. 1418-1427, 2001.

[68] J. F. Schnelle, P. G. MacRae, K. Giacobassi, H. S. H. MacRae, S. F. Simmons, and J. G. Ouslander, "Exercise with physically restrained nursing home residents: maximizing benefits of restraint reduction," Journal of the American Geriatrics Society, vol. 44, no. 5, pp. 507-512, 1996.

[69] C. D. Mulrow, M. B. Gerety, D. Kanten et al., "A randomized trial of physical rehabilitation for very frail nursing home residents," Journal of the American Medical Association, vol. 271, no. 7, pp. 519-524, 1994.

[70] Z. F. Kapasi, J. G. Ouslander, J. F. Schnelle, M. Kutner, and J. L. Fahey, "Effects of an exercise intervention on immunologic parameters in frail elderly nursing home residents," Journals of Gerontology A, vol. 58, no. 7, pp. 636-643, 2003.

[71] E. E. Baum, D. Jarjoura, A. E. Polen, D. Faur, and G. Rutecki, "Effectiveness of a group exercise program in a long-term care facility: a rondomized pilot trial," Journal of the American Medical Directors Association, vol. 4, no. 2, pp. 74-80, 2003.

[72] D. H. Sullivan, P. K. Roberson, E. S. Smith, J. A. Price, and M. M. Bopp, "Effects of muscle strength training and megestrol acetate on strength, muscle mass, and function in frail older people," Journal of the American Geriatrics Society, vol. 55, no. 1, pp. 20-28, 2007.

[73] D. H. Sullivan, P. K. Roberson, L. E. Johnson et al., "Effects of muscle strength training and testosterone in frail elderly males," Medicine and Science in Sports and Exercise, vol. 37, no. 10, pp. 1664-1672, 2005.

[74] E. Rydwik, E. Lammes, K. Frändin, and G. Akner, "Effects of a physical and nutritional intervention program for frail elderly people over age 75. A randomized controlled pilot treatment trial," Aging Clinical and Experimental Research, vol. 20, no. 2, pp. 159-170, 2008.

[75] J. L. Helbostad, O. Sletvold, and R. Moe-Nilssen, "Home training with and without additional group training in physically frail old people living at home: effect on healthrelated quality of life and ambulation," Clinical Rehabilitation, vol. 18, no. 5, pp. 498-508, 2004.

[76] K. Hauer, B. Rost, K. Rütschle et al., "Exercise training for rehabilitation and secondary prevention of falls in geriatric patients with a history of injurious falls," Journal of the American Geriatrics Society, vol. 49, no. 1, pp. 10-20, 2001.

[77] S. Vestergaard, C. Kronborg, and L. Puggaard, "Home-based video exercise intervention for community-dwelling frail older women: a randomized controlled trial," Aging Clinical and Experimental Research, vol. 20, no. 5, pp. 479-486, 2008.

[78] M. Bonnefoy, C. Cornu, S. Normand et al., "The effects of exercise and protein-energy supplements on body composition and muscle function in frail elderly individuals: a long-term controlled randomised study," British Journal of Nutrition, vol. 89, no. 5, pp. 731-738, 2003.

[79] M. E. T. McMurdo and L. Rennie, "A controlled trial of exercise by residents of old people's homes," Age and Ageing, vol. 22, no. 1, pp. 11-15, 1993.

[80] M. J. Faber, R. J. Bosscher, A. Chin, M. J. Paw, and P. C. van Wieringen, "Effects of exercise programs on falls and mobility in frail and pre-frail older adults: a multicenter randomized controlled trial," Archives of Physical Medicine and Rehabilitation, vol. 87, no. 7, pp. 885-896, 2006. 
[81] F. Landi, A. Russo, and R. Bernabei, "Physical activity and behavior in the elderly: a pilot study," Archives of Gerontology and Geriatrics. Supplement, no. 9, pp. 235-241, 2004.

[82] O. Seynnes, M. A. F. Singh, O. Hue, P. Pras, P. Legros, and P. L. Bernard, "Physiological and functional responses to lowmoderate versus high-intensity progressive resistance training in frail elders," Journals of Gerontology A, vol. 59, no. 5, pp. 503-509, 2004.

[83] H. Bruunsgaard, E. Bjerregaard, M. Schroll, and B. K. Pedersen, "Muscle strength after resistance training is inversely correlated with baseline levels of soluble tumor necrosis factor receptors in the oldest old," Journal of the American Geriatrics Society, vol. 52, no. 2, pp. 237-241, 2004.

[84] I. Bautmans, E. Van Hees, J. C. Lemper, and T. Mets, "The feasibility of whole body vibration in institutionalised elderly persons and its influence on muscle performance, balance and mobility: a randomised controlled trial," BMC Geriatrics, vol. 5, article 17, p. 8, 2005.

[85] C. Blanc-Bisson, A. Dechamps, G. Gouspillou, P. Dehail, and I. Bourdel-Marchasson, "A randomized controlled trial on early physiotherapy intervention versus usual care in acute car unit for elderly: potential benefits in light of dietary intakes," Journal of Nutrition, Health and Aging, vol. 12, no. 6, pp. 395399, 2008.

[86] Y. Mitani, K. Doi, T. Yano et al., "Effect of exercise using a horse-riding simulator on physical ability of frail seniors," Journal of Physical Therapy Science, vol. 20, no. 3, pp. 177-183, 2008.

[87] H. Shimada, S. Obuchi, T. Furuna, and T. Suzuki, "New intervention program for preventing falls among frail elderly people: the effects of perturbed walking exercise using a bilateral separated treadmill," American Journal of Physical Medicine and Rehabilitation, vol. 83, no. 7, pp. 493-499, 2004.

[88] H. Shimada, Y. Uchiyama, and S. Kakurai, "Specific effects of balance and gait exercises on physical function among the frail elderly," Clinical Rehabilitation, vol. 17, no. 5, pp. 472-479, 2003.

[89] D. Sato, K. Kaneda, H. Wakabayashi, and T. Nomura, "The water exercise improves health-related quality of life of frail elderly people at day service facility," Quality of Life Research, vol. 16, no. 10, pp. 1577-1585, 2007.

[90] M. Makita, H. Nakadaira, and M. Yamamoto, "Randomized controlled trial to evaluate effectiveness of exercise therapy (Takizawa Program) for frail elderly," Environmental Health and Preventive Medicine, vol. 11, no. 5, pp. 221-227, 2006.

[91] S. R. Lord, S. Castell, J. Corcoran et al., "The effect of group exercise on physical functioning and falls in frail older people living in retirement villages: a randomized, controlled trial," Journal of the American Geriatrics Society, vol. 51, no. 12, pp. 1685-1692, 2003.

[92] N. K. Latham, C. S. Anderson, A. Lee, D. A. Bennett, A. Moseley, and I. D. Cameron, "A randomized, controlled trial of quadriceps resistance exercise and vitamin D in frail older people: the frailty interventions trial in elderly subjects (FITNESS)," Journal of the American Geriatrics Society, vol. 51, no. 3, pp. 291-299, 2003.

[93] C. M. O’Hagan, D. M. Smith, and K. L. Pileggi, "Exercise classes in rest homes: effect on physical function," The New Zealand Medical Journal, vol. 107, no. 971, pp. 39-40, 1994.

[94] K. V. Hruda, A. L. Hicks, and N. McCartney, "Training for muscle power in older adults: effects on functional abilities," Canadian Journal of Applied Physiology, vol. 28, no. 2, pp. 178$189,2003$.
[95] D. A. Lazowski, N. A. Ecclestone, A. M. Myers et al., "A randomized outcome evaluation of group exercise programs in long-term care institutions," Journals of Gerontology A, vol. 54, no. 12, pp. M621-M628, 1999.

[96] L. Timonen, T. Rantanen, E. Mäkinen, T. E. Timonen, T. Törmäkangas, and R. Sulkava, "Effects of group-based exercise program on functional abilities in frail older women after hospital discharge," Aging Clinical and Experimental Research, vol. 18 , no. 1 , pp. 50-56, 2006.

[97] C. L. Rice and D. A. Cunningham, "Aging of the neuromuscular system: influences of gender and physical activity," in Gender, Physical Activity, and Aging, R. J. Shephard, Ed., pp. 121-150, CRC Press LLC, Boca Raton, Fla, USA, 2002. 


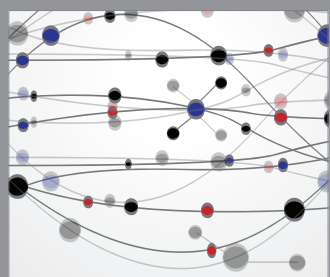

The Scientific World Journal
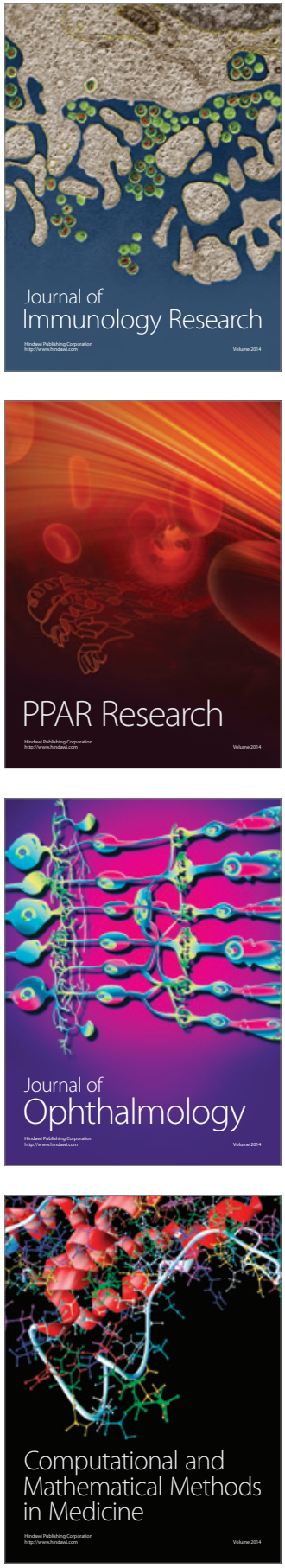

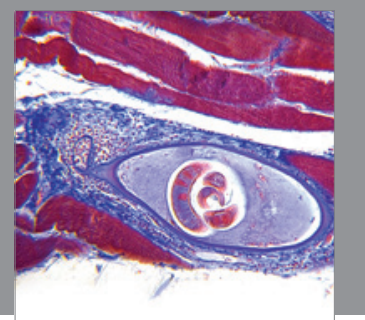

Gastroenterology

Research and Practice
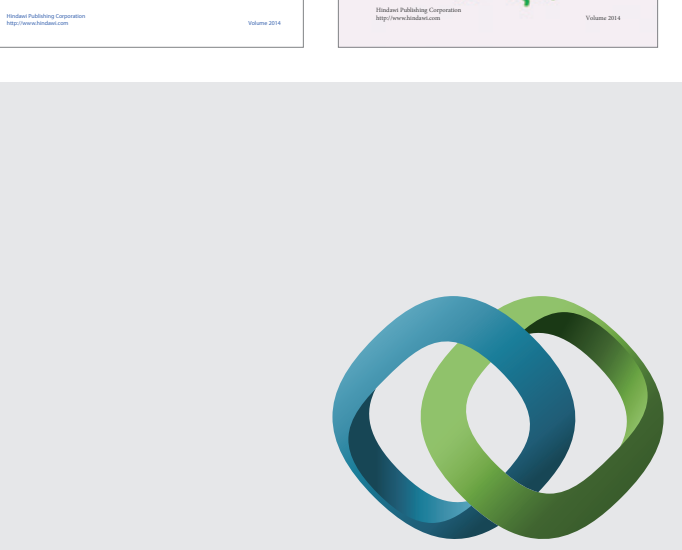

\section{Hindawi}

Submit your manuscripts at

http://www.hindawi.com
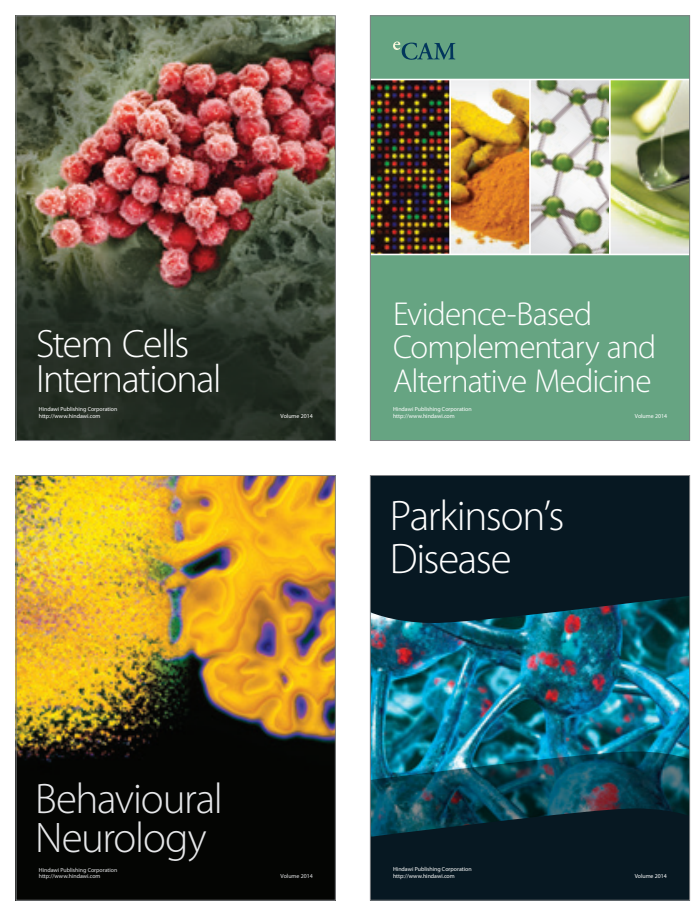

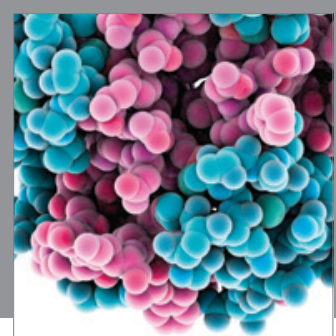

Journal of
Diabetes Research

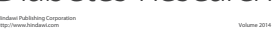

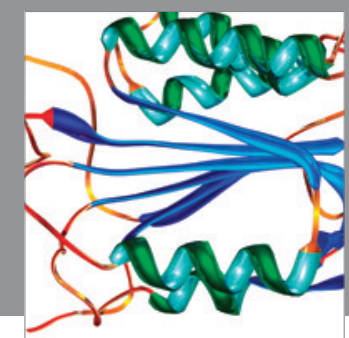

Disease Markers
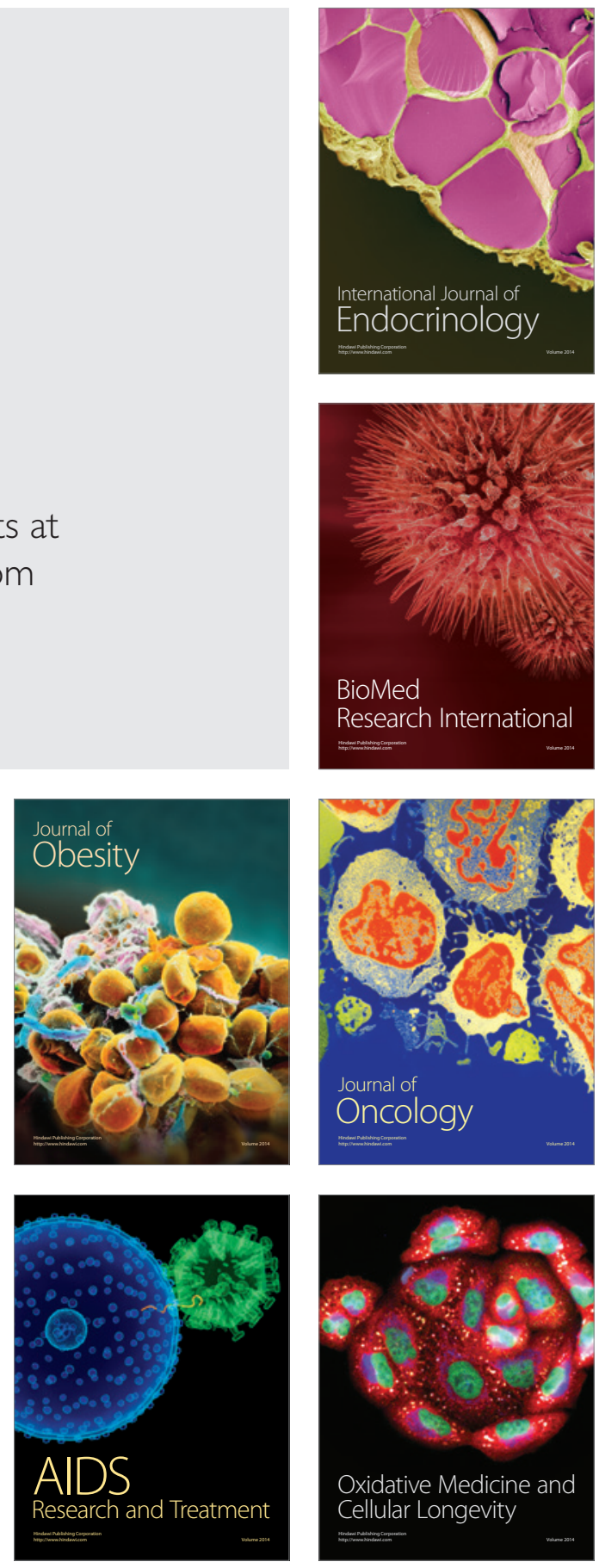